Submitted to the Astrophysical Journal

\title{
The Calán-Yale Deep Extragalactic Research (CYDER) Survey: Optical Properties and Deep Spectroscopy of Serendipitous X-ray Sources $^{1}$
}

\author{
Ezequiel Treister ${ }^{1,2,3}$, Francisco J. Castander ${ }^{4}$, Thomas J. Maccarone ${ }^{5}$, Eric Gawiser ${ }^{1,3,6}$, \\ Paolo S. Coppi ${ }^{1}$, C. Megan Urry ${ }^{2}$, José Maza ${ }^{3}$, David Herrera ${ }^{1}$, Valentino Gonzalez ${ }^{3}$, \\ Carlos Montoya ${ }^{3}$ and Pedro Pineda ${ }^{3}$ \\ treister@astro.yale.edu
}

\begin{abstract}
We present the first results from the Calán-Yale Deep Extragalactic Research (CYDER) survey. The main goal of this survey is to study serendipitous X-ray sources detected by Chandra in an intermediate flux range $\left(10^{-15}-\right.$ $10^{-12} \mathrm{ergs} \mathrm{s}^{-1}$ ) that comprises most of the X-ray background. $267 \mathrm{X}$-ray sources spread over 5 archived fields were detected. The $\log N-\log S$ distribution obtained for this sample is consistent with the results of other surveys. Deep $V$ and $I$ images were taken of these fields in order to calculate X-ray-to-optical flux ratios. Identifications and redshifts were obtained for 106 sources using optical spectroscopy from 8-m class telescopes to reach the optically faintest sources, to the same level as deeper X-ray fields like the Chandra Deep Fields, showing that the nature of sources detected depends mostly on the optical limit for spectroscopy. In general, sources optically classified as obscured Active Galactic Nuclei (AGNs) have redder optical colors than unobscured AGN. A rough correlation between $f_{X} / f_{\text {opt }}$ and hard X-ray luminosity was found for obscured
\end{abstract}

\footnotetext{
${ }^{1}$ Department of Astronomy, Yale University, P.O. Box 208101,New Haven,CT 06520.

${ }^{2}$ Yale Center for Astronomy \& Astrophysics, Yale University, P.O. Box 208121, New Haven, CT 06520

${ }^{3}$ Departamento de Astronomía, Universidad de Chile, Casilla 36-D, Santiago, Chile.

${ }^{4}$ Institut d'Estudis Espacials de Catalunya/CSIC,Gran Capità 2-4, E-08034 Barcelona, Spain.

${ }^{5}$ Astronomical Institute "Anton Pannekoek",University of Amsterdam,1098 SJ Amsterdam, The Netherlands
}

${ }^{6}$ NSF Astronomy and Astrophysics Postdoctoral Fellow 
AGN confirming the prediction by existing models that in obscured AGN the optical light is completely dominated by the host galaxy. The previously claimed decrease of the obscured to unobscured AGN ratio with increasing X-ray luminosity is observed. However, this correlation can be explained as a selection effect caused by the lower optical flux of obscured AGN. Comparison between the observed $N_{H}$ distribution and predictions by existing models shows that the sample appears complete up to $N_{H}<3 \times 10^{22} \mathrm{~cm}^{-2}$, while for more obscured sources incompleteness plays an important role in the observed obscured to unobscured AGN ratio.

Subject headings: galaxies: active, quasars: general, X-rays: galaxies,diffuse background

\section{Introduction}

Wide-area X-ray surveys have played a key role in understanding the nature of the sources that populate the X-ray universe. Early surveys like the Einstein Medium Sensitivity Survey (Gioia et al. 1990), ROSAT (Roentgen Satellite) International X-ray/Optical Survey (Ciliegi et al. 1997) and the ASCA (Advanced Satellite for Cosmology and Astrophysics) Large Sky Survey (Akiyama et al. 2000) showed that the vast majority of the X-ray sources were AGN. In particular, in shallow wide area surveys in the soft (0.5-2 keV) X-ray band, most of the sources detected are unobscured, broad line AGN, which are characterized by a soft X-ray spectrum with a photon index ${ }^{2} \Gamma=1.9$ (Nandra \& Pounds 1994).

More recent, deeper observations, mostly by ROSAT (Hasinger et al. 1998), XMMNewton and Chandra (Rosati et al. 2002), that resolved between $70 \%$ and $90 \%$ of the $\mathrm{X}$-ray background (XRB) showed that the vast majority of this background radiation can be attributed to AGN. However, the spectrum of the XRB is well characterized up to $E \sim 30 \mathrm{keV}$ by a power law with photon index $\Gamma=1.4$ (Gruber et al. 1999), harder than the typical unobscured AGN spectrum (Mushotzky et al. 2000). Given that photoelectric extinction preferentially absorbs soft X-ray photons (Morrison \& McCammon 1983), the X-ray spectra of obscured AGN look harder and therefore more compatible with the observed spectral

\footnotetext{
${ }^{1}$ Partly based on observations collected at the European Southern Observatory, Chile, under programs 68.A-0459 and 72.A-0509

${ }^{2}$ We define the photon index $\Gamma$ as the exponent giving a photon flux density X-ray spectrum $d N / d E \propto E^{-\Gamma}$ in photons $\mathrm{cm}^{-2} \mathrm{~s}^{-1} \mathrm{keV}^{-1}$
} 
shape of the XRB. Therefore, population synthesis models (Madau et al. 1994; Comastri et al. 1995; Gilli et al. 1999, 2001) that can explain the spectral shape and normalization of the XRB use a combination of obscured and unobscured AGN as the major contributor. In these models, the ratio of obscured to unobscured AGN is about 4:1 (Gilli et al. 2001) with a redshift peak at $z \sim 1.3$. However, recent deep optical spectroscopic follow up in the Chandra Deep Fields (CDF) North (Barger et al. 2003) and South (Szokoly et al. 2004) revealed a much lower redshift peak at $z \sim 0.8$ and an obscured to unobscured AGN ratio of $\sim 2: 1$.

While large observational efforts have been concentrated in the Chandra Deep Fields, which provide the deepest view of the X-ray Universe (e.g., a flux limit of $\simeq 2.5 \times 10^{-17} \mathrm{ergs} \mathrm{cm}^{-2} \mathrm{~s}^{-1}$ on the CDF-N), the small area covered $\left(\simeq 0.07 \mathrm{deg}^{2}\right.$ each $)$ does not allow them to obtain a statistically significant number of sources in the intermediate X-ray flux range $\left(10^{-15}-10^{-12}\right.$ ergs $\mathrm{cm}^{-2} \mathrm{~s}^{-1}$ ) that contributes $\sim 60-70 \%$ of the XRB. Therefore, we obtained identifications and studied the multiwavelength properties of X-ray sources in this flux range over a much larger area.

Specifically, in 2001 we started the Calán-Yale Deep Extragalactic Research (CYDER) survey, a multiwavelength study of serendipitous X-ray sources in existing, archived, moderately deep Chandra fields. Initial results from the first two fields studied were presented by Castander et al. (2003b). Also, two high-redshift $(z>4)$ X-ray selected quasars discovered in this survey, a significant fraction of the total sample known today $(\sim 10)$, were reported by Castander et al. (2003a) and Treister et al. (2004a). Near infrared images in the $J$ and $K$ bands were obtained for these fields up to $J \sim 21$ and $K \sim 20$ mags (Vega). The results of combining X-ray/optical and near infrared observations for our sample of serendipitous $\mathrm{X}$-ray sources will be presented in a following paper (F. Castander et al, in prep).

In this paper, we present optical photometry for $267 \mathrm{X}$-ray sources selected in the Chandra total band (0.5-8 keV) in the five fields studied by the CYDER survey. Also, spectroscopic identifications and redshifts for $106 \mathrm{X}$-ray sources are presented. The sample presented here is comparable in multiwavelength follow-up to deeper, more famous, surveys like the CDFs and the Lockman Hole. Spectroscopic identifications were obtained for sources with relatively faint optical fluxes ( $V \sim 24 \mathrm{mag}$ ), allowing for a more unbiased study of the $\mathrm{X}$ ray population and showing that the statistical properties of the sample depends significantly on the depth of the spectroscopic follow-up. Also, the use of five different fields spread over the sky allows to reduce the effects of cosmic variance, that affected the results of single-field studies, e.g., the presence of clusters in the CDF-S (Gilli et al. 2003).

In $\S 2$ we explain the criteria used to select the X-ray fields studied and the procedures followed to reduce the X-ray data and to extract sources lists. In $\S 3$ we describe the optical 
imaging and spectroscopy observations and the data reduction methods used. In $\S 4$ we present source properties in each wavelength range. Our results are discussed in $\S 5$ and the conclusions outlined in $\S 6$. Throughout this paper we assume $H_{o}=70 \mathrm{kms}^{-1} \mathrm{Mpc}^{-1}$, $\Omega_{m}=0.3$ and $\Omega_{\Lambda}=0.7$, consistent with the cosmological parameters reported by Spergel et al. (2003).

\section{X-ray Data}

\subsection{Field Selection}

Fields in the CYDER survey were selected based on existing deep Chandra observations available in the public archive before the optical imaging campaign started in late 2000 . The fields are observable from the southern hemisphere, are at high galactic latitude $\left(|b|>40^{\circ}\right)$ in order to minimize dust extinction, avoiding targeting known clusters given the difficulties in dealing with a diffuse non-uniform background. Only observations with the Advanced CCD Imaging Spectrometer (ACIS; Garmire et al. 2003) were used. In the case of ACIS-I observations all four CCDs were used while for observations in the ACIS-S mode only the S3 and S4 chips were used in order to keep the off-axis angle small and therefore only use zones with good sensitivity. Fields selected for this study are presented in Table 1. In two of these fields, C2 and D2, the original target of the observation was a galaxy group. In these cases, the galaxy group diffuse emission reduces the sensitivity in the centers of the regions, but not dramatically. Roughly the central 40" radius region for HCG 62 has substantial gas emission, while the $1^{\prime}$ central region was affected by the presence of HCG 90. This accounts for about $2 \%$ of the HCG 62 region and a slightly smaller fraction of the HCG 90 region, since the latter was observed with ACIS-I. The effective area of field C5 was set to 0 since the Chandra images of that field were read in subraster mode to include only the central source, and therefore serendipitous sources detected on that field were ignored to compute the $\log N-\log S$ relation. Some of our fields were also studied by other similar surveys. In particular, the Q2345 and SBS 0335 fields were analyzed by the Chandra Multiwavelength Project (ChaMP; Kim et al. 2004) while the HCG 62 and Q2345 fields were studied by the Serendipitous Extragalactic X-ray Source Identification Program (SEXSI; Harrison et al. 2003). 


\subsection{X-ray Data Reduction}

Reduction of the data included the removal of bad columns and pixels using the guidelines specified on the "ACIS Recipes: Clean the Data" web page and the removal of flaring pixels using the FLAGFLARE routine. We used the full set of standard event grades $(0,2,3,4,6)$ and created two images, one from 0.5 to $2.0 \mathrm{keV}$ and one from 2.0 to $8.0 \mathrm{keV}$. Then, we used the WAVDETECT routine from the CIAO package to identify the point sources within these images, checking wavelet scales 1,2,4,8 and 16. Sources were extracted independently in the soft $(0.5-2.0 \mathrm{keV})$ and hard $(2.0-8.0 \mathrm{keV})$ band images. The false source detection probability is set to $10^{-6}$ for ACIS-S observations and $10^{-7}$ for ACIS-I observations. This gives a likelihood of $\sim 1$ false source detection per field observed. Given the low density of X-ray sources and the good spatial resolution of Chandra, matching sources in the soft and hard bands was straightforward.

Where the X-ray spectrum had at least 60 counts, the photons were binned in groups of 20, and the spectrum was fit in XSPEC 11.0, using a model consisting of a power law with the appropriate Galactic absorption value for each field. Where the number of counts was smaller than 60, the same procedure was used, except that the spectral index was fixed to $\Gamma=1.7$, consistent with the hardening of the X-ray spectrum with decreasing flux (Giacconi et al. 2001).

\section{Optical Data}

\subsection{Optical Imaging}

Optical images were obtained using the CTIO 4-m Blanco Telescope in Chile with the MOSAIC-II camera (Muller et al. 1998), which has a field of view of $36^{\prime} \times 36^{\prime}$. Details of the optical observations are presented in Table 2. All the fields have optical coverage in the $V$ and $I$ filters in the optical, and were also imaged in the $J$ and $K$ bands in the near infrared, observations that will be reported in a following publication.

Reduction of the data was performed using standard procedures included in IRAF $\mathrm{v}$ $2.12^{3}$, in particular in the MSCRED package. The data reduction scheme followed was

\footnotetext{
${ }^{3}$ IRAF is distributed by the National Optical Astronomy Observatory, which is operated by the Association of Universities for Research in Astronomy, Inc., under cooperative agreement with the National Science Foundation.
} 
based on the recipe used by the NOAO Deep Wide Survey ${ }^{4}$. Standard calibration images (bias and dome flats) were obtained each night for every filter used. Super-sky flats were constructed based on several $(\sim 20)$ unregistered frames in each filter, masking real objects in order to obtain a secondary flat field image. Once basic calibrations were performed, individual frames were registered and co-added to obtain the final image in each filter. Astrometric solutions for each final image were calculated based on the USNO catalog. Typical astrometric uncertainties are $\sim 0.3$ ", smaller than the on-axis PSF size of Chandra images, therefore allowing for an accurate match between optical and X-ray data.

Objects in the final images were extracted using SExtractor (Bertin \& Arnouts 1996). To detect objects we used a threshold of $1.5 \sigma$ above the background per pixel and a minimum area of 15 pixels $\left(\sim 1.0 \operatorname{arcsec}^{2}\right)$ above that threshold. In several experiments, this combination of parameters gave a good balance between completeness and false detections, the latter being lower than $\sim 5 \%$, for the range of FWHM of our images.

Zero points for each image were obtained independently for each night based on observations of Landolt standard fields (Landolt 1992). Aperture photometry was then performed using a diameter of 1.4 times the FWHM. Magnitudes were later corrected for the (small) effects of Galactic extinction in our high-galactic latitude fields. Limiting magnitude for each image was calculated based on global RMS measurements of the background and reported in Table 2.

Objects in the $V$ and $I$ images were matched by position allowing a maximum distance of $1^{\prime \prime}$ between objects in different filters. The typical difference between the $V$ and $I$ counterparts is $\sim 0.5^{\prime \prime}$, consistent with the previously reported astrometric uncertainties and typical centroid errors, so the choice of $1^{\prime \prime}$ as a threshold provides a good balance in order to avoid spurious matches. If more than one match was found inside that area then the closest match was used, however this only happened in a few cases given the typical sky density of our optical images. $V-I$ color was calculated for sources detected in both bands.

\subsection{Optical Spectroscopy}

Given that one of the goals of CYDER is the study of the optically faint X-ray population, only 8-m class telescopes were used for the spectroscopic follow-up. Multi-object spectrographs were used in order to improve the efficiency of the observations, including

\footnotetext{
${ }^{4}$ The data reduction cookbook and a description of the survey design can be found in the web page http://www.noao.edu/noao/noaodeep/ReductionOpt/frames.html
} 
FORS2 at the VLT in MXU mode and the LDSS-2 instrument on the Magellan I (Baade) Telescope. Details of these observations are given in Table 3.

Given the space density of X-ray sources at our flux limit and the field of view of the instruments used, typically $\sim 8 \mathrm{X}$-ray sources were observed per mask. For the observations with FORS2 at the VLT, the 300V-20 grism was used, which gives a resolution $R \sim 520$ $(10.5 \AA)$ for our 1 " slits, with a typical wavelength coverage from $4000-9000 \AA$ depending on the position of the source in the mask. Observations with LDSS-2 used the Med/Blue grism, giving a dispersion of $5.3 \AA$ pixel $^{-1}$ at a central wavelength of $5500 \AA$ and resolution of $R \sim 350$ with our 1.0" wide slits. The typical wavelength coverage with this configuration was $\sim 4000-7500 \AA$.

Spectral reduction was performed using standard IRAF tasks called by a customized version of the BOGUS code $^{5}$. We calibrated the wavelength of the spectrum using HeAr comparison lamps and the night-sky lines. In order to flux-calibrate our spectra, $\sim 2$ spectrophotometric standards were observed every night.

\subsection{Catalog}

The full catalog of X-ray sources in the CYDER field is presented in the on-line version of the journal, while for clarity a fraction of the catalog is presented in Table 4. The full catalog is also available on-line at http://www.astro.yale.edu/treister/cyder/. Coordinates are given as measured in the X-ray image, while the offset is calculated with respect to the closest optical counterpart and only reported when this offset is smaller than 2.5" and thus that counterpart was used in the analysis. When a counterpart was not detected in the optical images, the $5 \sigma$ upper limit in that band is reported. In order to convert count rates into fluxes, the procedure described in section $\S 2.2$ was followed. The observed X-ray luminosity was computed only for sources with spectroscopic identification and measured redshift. This luminosity was calculated in the observed frame without accounting for $\mathrm{k}$ corrections or correcting for absorption. Therefore, the simple formula $L_{X}=4 \pi d^{2} f_{X}$ was used.

\footnotetext{
${ }^{5}$ Available at http://zwolfkinder.jpl.nasa.gov/ stern/homepage/bogus.html
} 


\section{Source Properties}

\subsection{X-ray}

A total of $37 \mathrm{X}$-ray sources were detected in the $\mathrm{C} 2$ field, with a total $(0.5-8 \mathrm{keV}) \mathrm{X}$-ray flux ranging from $1.7 \times 10^{-15}$ to $5 \times 10^{-13} \mathrm{ergs}^{-2} \mathrm{~s}^{-1}$. In the $\mathrm{C} 5$ field only $5 \mathrm{X}$-ray sources were detected above $1.6 \times 10^{-14} \mathrm{ergs}^{-2} \mathrm{~cm}^{-1}(0.5-8 \mathrm{keV})$ since only the s3 CCD was read and in subraster mode. In the D1, D2 and D3 fields 93, 47 and $85 \mathrm{X}$-ray sources were detected respectively, with a total $(0.5-8 \mathrm{keV}) \mathrm{X}$-ray flux ranging from $8 \times 10^{-16}$ to $1 \times 10^{-13} \operatorname{ergs~cm}^{-2} \mathrm{~s}^{-1}$.

The area covered as a function of limiting flux in the hard X-ray flux band was first estimated individually for each field using the Portable, Interactive Multi-Mission Simulator (PIMMS; Mukai 1993). Given the complexities associated with the modeling of the varying PSF as a function of off-axis angle and the presence of diffuse emission in most of these fields that makes the problem of estimating the completeness levels even harder a constant, higher, flux limit was used for each field. Specifically, for each field we used a fixed value of 2.5 times the flux of the faintest source included in the catalog for each field, in order to be sure that the sample is complete up to that flux. This roughly corresponds to 20 counts detected in the hard band for ACIS-S observations and 10 counts for fields observed with the ACIS-I CCDs. The flux limit in the hard band assumed for each field is shown on Table 1. The resulting total area of the survey is $\gtrsim 0.1 \mathrm{deg}^{-2}$, with a minimum flux limit of $1.3 \times 10^{-15} \mathrm{ergs} \mathrm{cm}^{-2} \mathrm{~s}^{-1}(2-8 \mathrm{keV})$. Figure 1 shows the resulting area versus flux limit curve, in comparison to other surveys like the Great Observatories Origin Deep Survey (GOODS; Giavalisco et al. 2004) and SEXSI (Harrison et al. 2003).

The cumulative flux distribution was calculated using

$$
N(>S)=\sum_{i ; S_{i}>S} \frac{1}{A_{i}\left(S_{i}\right)}
$$

where $S_{i}$ is the observed hard X-ray flux of the $i$-th source and $A_{i}$ is the maximum area over which that source could be detected. The resulting $\log N-\log S$ relation for the CYDER sample is shown in Figure 2. This curve is consistent with the relation computed by other authors (e.g., Moretti et al. 2003; Ueda et al. 2003). In the lower panel of Fig 2 we show the residuals after comparing with the relation computed by Moretti et al. (2003) using a combination of observational data in both shallow wide-field and deep pencil beam X-ray surveys, showing that the agreement is good.

One significant problem with the cumulative $\log N-\log S$ relation is that the errors in

each bin are not independent. The differential flux distribution can be used to avoid this 
problem. This relation can be expressed as:

$$
n(S)_{i}=\sum \frac{N_{i}}{\Delta S_{i} A_{i}\left(S_{i}\right)}
$$

where in this case the sample was binned with a bin size of $2 \times 10^{-15} \mathrm{erg} \mathrm{cm}^{-2} \mathrm{~s}^{-1}$. $N_{i}$ is the number of sources in the i-th bin, $\Delta S_{i}$ is the size of the bin and $A_{i}$ is the the total area over which sources in this bin could be detected. In Figure 3 the resulting differential $\log N-\log S$ is shown. These results were compared to the relation reported by Harrison et al. (2003), which fitted the SEXSI data with a broken power-law given by

$$
n(S)=(46.8 \pm 2.1)\left(\frac{S}{10^{-14}}\right)^{-2.46 \pm 0.08}
$$

for $S>1.25 \times 10^{-14}$ ergs $\mathrm{cm}^{-2} \mathrm{~s}^{-1}$ and

$$
n(S)=(43.65 \pm 2)\left(\frac{S}{10^{-14}}\right)^{-1.41 \pm 0.17}
$$

for fainter sources. As it is shown in the lower panel of Figure 3, this parametrization

provides a good fit to the CYDER data, even though some scatter is present. A $\chi^{2}$ test to this fit compared to the observed data gave a reduced $\chi^{2}$ of 1.37 .

\subsection{Optical}

207 of the total 267 X-ray sources $(77 \%)$ were detected in our optical $V$-band images, searching in a radius of $2.5^{\prime \prime}$ ( $\sim 4$ times the typical seeing) around the centroid of the X-ray emission. The average offset between an X-ray source and the nearest optical counterpart is $\sim 1.3^{\prime \prime}$ with a standard deviation of $\sim 0.5^{\prime \prime}$. In our optical images we detected typically $\sim 60,000$ sources in $30^{\prime} \times 30^{\prime}$ so the chance of having a random source in a $2.5^{\prime \prime}$ radius is $\sim 36 \%$ and therefore our choice of $2.5^{\prime \prime}$ as the maximum allowed offset is reasonable to avoid spurious associations. In cases where more than one counterpart was found inside this radius the closest optical source to the X-ray centroid was assumed to be the right counterpart. The $V$ magnitude distribution for the $\mathrm{X}$-ray sources with detected optical counterparts in the CYDER fields is shown on Figure 4. X-ray sources cover the range from $V \simeq 16$ to 26 mags and higher (fainter than our optical magnitude limit). The hatched histogram in Figure 4 shows the magnitude distribution of sources targeted for spectroscopy, while the cross-hatched histogram shows the distribution for sources with spectroscopic identifications. A K-S test performed comparing the total $V$ magnitude distribution to the magnitude distribution for sources targeted for spectroscopy revealed that the hypothesis that both distributions are drawn from the same parent distribution (the null hypothesis) is accepted at 
the $98.7 \%$ confidence level. However, the effect of the optical flux in the efficiency of spectroscopic identifications can be seen by comparing the magnitude distribution for sources successfully identified and the total sample, namely the incompleteness of the sample with spectroscopic identification at the faint optical flux end is evident in this figure, even though the target selection was independent of the optical properties of the source. This effect is also observed in the $I$-band (Figure 5), where $181 \mathrm{X}$-ray sources counterparts were detected $(68 \%)$, a lower number than in the deeper $V$-band images. In this case, the decrease in the efficiency of spectroscopic identifications with decreasing optical flux is also evident in Figure 5. The average $V-I$ color for X-ray sources with optical counterparts is 0.92 ; its distribution, shown in Figure 6, shows that the efficiency of the spectroscopic identifications is independent of the $V-I$ color of the optical counterpart. A K-S test performed comparing the sample with spectroscopic identification to the total sample show that both distributions are drawn from the same parent distribution with a confidence level of $99.89 \%$.

53 out of $267(\sim 20 \%)$ sources were not detected in any of the two optical bands. The vast majority of these sources are also very faint in X-rays, so that many of them were only detected in the soft band, that is more sensitive in Chandra. This does not imply that they have an intrinsically soft spectrum. In fact, given the known relation between hardness ratio and X-ray flux (Giacconi et al. 2001), it is plausible that these sources are hard and therefore good candidates to be obscured AGN. 5 of these sources were only detected in the hard band, and therefore should have a very hard spectrum in X-rays, that combined with the fact that are very faint in the optical bands makes them good candidates to be obscured AGN at relatively high redshifts. This lack of detection of X-ray faint sources in optical images acts as a selection effect against the study of obscured AGN. However, this bias can be overcome by studying these sources in the near infrared (Gandhi, Crawford, Fabian, \& Johnstone 2004), where the effects of dust obscuration are much smaller. In a following paper (F. Castander, in prep) properties of these sources in the near infrared bands will be presented.

The $V$-band magnitude versus redshift plot (Figure 7) reveals how the source composition changes with redshift, and how it is potentially affected by the implicit optical flux cut for spectroscopy. While at low redshift $(z<1)$ we find mostly obscured AGN and normal galaxies, characterized by an absolute optical magnitude $M_{V} \gtrsim-21$, at higher redshift they become too faint in the optical bands and therefore only unobscured AGN (that have mostly $\left.M_{V}<-22\right)$ can be found. This implies that our survey may be biased against detecting obscured AGN at high redshift. This effect is investigated in more detail in $\S 5$. 


\subsection{Correlations}

Sources with spectroscopic identification were classified using a combination of optical and X-ray criteria. X-ray sources showing stellar spectra were classified as stars. For extragalactic sources, the X-ray luminosity was computed from the observed X-ray flux using the relation

$$
L_{X}=4 \pi d_{L}^{2} f_{X}
$$

where $d_{L}$ is the luminosity distance calculated for the assumed cosmology. This luminosity is therefore the uncorrected, observed frame X-ray luminosity. No attempt was made to correct for dust obscuration or k-corrections given that for most sources the number of observed counts was too small to perform spectral fitting and therefore to calculate the neutral hydrogen column density $N_{H}$ or the intrinsic spectral shape.

In order to separate X-ray emission generated by AGN activity from the emission coming from X-ray binaries and star formation in galaxies we used a simple X-ray luminosity threshold criterion. Locally, the most X-ray luminous star forming galaxy known (NGC 3256) has a total X-ray luminosity $L_{X} \simeq 8 \times 10^{41} \mathrm{ergs} \mathrm{s}^{-1}$ in the (0.5-10) keV band (Lira et al. 2002). Another source of luminous X-ray emission is the presence of hot gas in elliptical galaxies, which at low redshift is extended and therefore easily separated from AGN emission; at high redshift it is not resolved and thus harder to separate from AGN activity. However, according to the O'Sullivan et al. (2001) catalog of elliptical galaxies with detected X-ray emission, only a few normal galaxies have $L_{X}>10^{42} \mathrm{ergs} \mathrm{s}^{-1}$. Therefore, we adopted $L_{X}=10^{42} \mathrm{ergs} \mathrm{s}^{-1}$ in the total $(0.5-8 \mathrm{keV})$ band as the threshold separating sources dominated by AGN activity from those dominated by star formation or other processes in a galaxy. Given the relatively low number of galaxies found in the survey, we expect this classification method to have a small effect on the total numbers of AGN reported.

Objects with a total X-ray luminosity $L_{X}<10^{42} \mathrm{ergs} \mathrm{s}^{-1}$ and narrow emission or absorption lines (velocity dispersion less than $1000 \mathrm{~km} \mathrm{~s}^{-1}$ ) were classified as normal galaxies, while sources with $L_{X}>10^{42} \mathrm{ergs} \mathrm{s}^{-1}$ were classified as unobscured (type 1) or obscured (type 2) AGN depending on whether they show broad or narrow lines on their optical spectrum. Furthermore, sources with $L_{X}>10^{44} \mathrm{ergs} \mathrm{s}^{-1}$ are called QSO-1, or simply QSO, if they have broad lines or QSO-2 if the lines are narrow, but they are still considered AGN.

For sources with spectroscopic identification, Figure 8 shows the $V-I$ color as a function

of redshift. X-ray sources identified as Type 1 AGN (broad emission lines) fall near the position expected for QSOs, calculated convolving the optical filters with the Sloan Digital Survey Composite Quasar Spectrum (Vanden Berk et al. 2001). Galaxies/Type 2 AGN, which are only detected up to $z \sim 1.5$, are located in the region expected for galaxies ranging 
from Elliptical to Sb types and have redder colors than Type 1 AGN. The expected colors for each type of galaxy at a given redshift were calculated using the galaxy spectrum models of Fioc \& Rocca-Volmerange (1997) assuming that there is no evolution in the spectrum with redshift. From Figure 8 it is clear that objects classified as obscured AGN have redder colors, consistent with those of the host-galaxies. In fact, obscured AGN have an average $V-I$ color of 1.46 with a standard deviation of 0.58 , while unobscured AGN have an average color of 0.56 and standard deviation of 0.46

The redshift distribution for sources with spectroscopic identification is shown in Figure 9. When the whole sample of X-ray sources is considered, this distribution has a maximum at very low redshift, $z \simeq 0-0.6$. However, when only sources with $L_{X}>10^{42} \mathrm{ergs} \mathrm{s}^{-1}$ (i.e., those dominated by AGN activity) are included the peak is displaced to higher redshifts, namely $z \sim 1$. As it is shown by the hatched distribution in Figure 9 , the high redshift population $(z>1.3)$ is completely dominated by broad line AGN (most of them quasars with $L_{X}>10^{44} \mathrm{ergs} \mathrm{s}^{-1}$ ). This is explained by the high optical luminosity of these objects, which makes them easier to identify, even at large distances and by the lack of near infrared information at this point, that is very useful to detect obscured AGN, in particular at high redshift (Gandhi, Crawford, Fabian, \& Johnstone 2004).

In order to investigate possible relations between X-ray and optical emission for different classes of sources, in Figure 10 we plot hard X-ray flux versus $V$-band magnitude. Most of the sources are located in the region bounded by $\log f_{X} / f_{\text {opt }}= \pm 1$. Starburst galaxies detected in X-rays are typically bright in the optical bands and faint in X-rays, and are therefore characterized by $\log f_{X} / f_{\text {opt }}<-1$ (see Hornschemeier et al. (2003) and references therein). Unobscured (type 1) AGN/Quasars are located around the $\log f_{X} / f_{\text {opt }}=1$ (Giacconi et al. 2002) position although the scatter is large, while obscured (type 2) AGN/Quasars have in general $\log f_{X} / f_{\text {opt }}>1$ since most of the optical light from the central engine is blocked from our view but low-luminosity examples of obscured AGN can be found also with $\log f_{X} / f_{\text {opt }} \simeq$ 0 as it will be discussed in $\S 5$. Unidentified sources at high $f_{X} / f_{\text {opt }}$ are unlikely to be unobscured AGN because their broad emission lines would have been easy to see in the optical spectra, so they are probably obscured AGN.

Figure 11 shows the observed-frame hard X-ray $(2-8 \mathrm{keV})$ luminosity versus redshift diagram for sources with spectroscopic redshifts. If we assume a flux limit of $6.9 \times 10^{-16} \mathrm{ergs} \mathrm{cm}^{-2} \mathrm{~s}^{-1}$ (which would yield a total of 5 counts in the $0.5-8 \mathrm{keV}$ band in a Chandra ACIS-I $60 \mathrm{ks}$ observation for $\Gamma=1.7$ ), the solid line in Figure 11 shows the detection limit for X-ray sources in our survey. If an optical magnitude of $V=25$ mag is taken as the approximate flux limit for spectroscopy (there are fainter sources for which spectroscopy is possible, but the identification relies in the presence of strong emission lines) and a ratio of X-ray to 
optical emission of $f_{X} / f_{\text {opt }}=1$ is assumed, then the dashed line in Figure 11 shows our limiting magnitude as a function of redshift for sources with spectroscopy. This explains why incompleteness of the spectroscopic sample is particularly important at high redshift, where the fraction of X-ray sources with spectroscopic identification declines.

If the same material that is causing the absorption of X-rays is responsible for the extinction in the optical bands, then a relation between these two quantities can be expected, namely the reddest sources in the optical (higher value of $V-I$ ) should also be the X-ray hardest sources. A typical way to quantify the steepness of the X-ray spectrum is using the hardness ratio (HR) defined as

$$
H R=\frac{H-S}{H+S}
$$

where $H$ and $S$ are the count rates in the hard and soft X-ray bands respectively.

In Figure 12 the $\mathrm{HR}$ versus $V-I$ optical color is presented. In this diagram, no clear relation between $\mathrm{HR}$ and optical color is observed. The absence of a correlation can be explained by the differences in the intrinsic optical and X-ray spectrum for different types of sources detected in the X-ray bands, independent of the amount of obscuration present. Note, however, that in general sources optically classified as obscured AGN are redder (larger $V-I$ colors) than unobscured AGN as was previously observed on Figure 8 and also tend to have higher values of HR. This lack of a strong relationship between HR and optical color even for sources classified as AGN-dominated can be explained in part by the effects of K-corrections caused by the different redshifts of the sources and by changes in the intrinsic spectrum with parameters other than obscuration, e.g. luminosity (Ho 1999). Sources without spectroscopic identification (crosses on Figure 12) have in general redder colors than unobscured AGN, that are similar to the colors of spectroscopically confirmed obscured AGN and therefore are consistent with being moderately obscured AGN at relatively high redshift $(z \gtrsim 1)$. Most of these sources however present a soft X-ray spectrum, that can be explained if these sources are at moderately high redshift so that the observed frame Chandra bands traces higher energy emission, that is less affected by absorption. However, this is highly speculative, and the final answer about the nature of these optically faint X-ray sources will come from either deeper optical spectroscopy or from the near infrared data.

\subsection{Identifications}

Of the 267 X-ray sources detected in the CYDER fields, 106 were identified using optical spectroscopy. While the fraction of sources identified is biased toward higher optical fluxes (see Figures 4 and 5), Figure 6 shows that the optical colors of the sources in the sample 
both targeted and identified by optical spectroscopy follow a similar distribution as those of the total sample.

The redshift distribution of the sample with spectroscopic identification is presented in Figure 9. X-ray sources in this sample span a wide range in redshift, $0<z<4$.6. The mean redshift for our extragalactic sample with spectroscopic identification is $\langle z\rangle=1.19$ and the peak is located at a low redshift, $z \simeq 0.2-0.6$. When only the sources dominated by AGN activity (i.e., $L_{X}>10^{42} \mathrm{ergs} \mathrm{s}^{-1}$ ) are considered, the mean redshift is $\langle z\rangle=1.34$ while the peak is at $z \simeq 0.5$. For sources optically classified as unobscured AGN, the average redshift is $\langle z\rangle=1.82$ and the peak is at $z_{p}=1.3$. Therefore, we conclude that the nature of the identified X-ray sources changes as a function of redshift. At $z<0.3$, the sample is dominated by normal galaxies $(\sim 60 \%)$ and obscured AGN. In the $0.3<z<1$ region, just a few normal galaxies are found and the population is dominated by obscured AGN (77\%), while at $z>1$ the vast majority of the sources found are unobscured AGN.

The hard X-ray luminosity distribution for the sample of sources with spectroscopic redshift can be seen in Figure 13. In terms of luminosity, the few sources optically classified as galaxies detected in the X-ray sample dominate the low luminosity bins. In the intermediate X-ray luminosity bins $\left(10^{42}<L_{X}<10^{44} \mathrm{ergs}^{-2} \mathrm{~s}^{-1}\right)$, most of the sources are optically classified as obscured AGN, while in the higher luminosity bins $\left(L_{X}>10^{44} \mathrm{ergs} \mathrm{cm}^{-2} \mathrm{~s}^{-1}\right)$ the vast majority of the sources are optically identified as unobscured AGN. This change of the source type as a function of X-ray luminosity is further investigated in $\S 5$.

In our sample there is only one source classified as QSO-2 based on its observed X-ray luminosity and optical spectrum: CXOCY-J125315.2-091424 at $z=1.154$, located in the $\mathrm{C} 2$ field. 52 counts were detected in the hard X-ray band, while no emission was detected in the soft X-ray band and therefore the hardness ratio is -1 . The optical spectrum of this source is presented in Figure 14. Narrow emission lines like CIII, MgII and OII are clearly visible in this spectrum and were used to calculate the redshift of the source. The total (0.5-8 $\mathrm{keV}$ ) observed X-ray luminosity of this source is $L_{X} \simeq 2 \times 10^{44} \mathrm{ergs} \mathrm{s}^{-1}$ making this the brightest obscured AGN in our sample. Given the observed hardness ratio and redshift of this source, the expected neutral hydrogen column density in the line of sight is $\gtrsim 10^{23} \mathrm{~cm}^{-2}$ assuming either an intrinsic power law with exponent 1.9 or 1.7 , consistent with the optical classification of very obscured AGN.

In the total sample with spectroscopic identification 7 sources are classified as stars (6.6\%), 11 as normal galaxies (10.4\%), 38 are identified as obscured AGN (35.8\%), and 50 as unobscured AGN (47.2\%). These fractions are similar to the findings of other X-ray surveys, as shown in Table 5. 
The ChaMP Survey (Kim et al. 2004) covers a total of $14 \mathrm{deg}^{2}$. In their first spectroscopy report, 6 Chandra fields were covered to a depth of $r \simeq 21$ mag (Green et al. 2004). In order the compare with their results, we applied our classification scheme to their data. Namely, narrow-line and absorption-line galaxies with $L_{X}>10^{42} \mathrm{ergs} \mathrm{s}^{-1}$ were classified as obscured AGN, sources with broad lines as unobscured AGN, while the remaining extragalactic sources were classified as galaxies. The main reason for the discrepancies between their source mix and ours (Table 5 ) is the optical magnitude cut for spectroscopy, $\sim 2$ magnitudes brighter than CYDER, which explains why their sample is clearly dominated by unobscured AGN, the optically brighter X-ray emitting sources.

In Table 5, our sample is also compared to both the Chandra Deep Fields North (Brandt et al. 2001) and South (Giacconi et al. 2002), each covering $\sim 0.1 \mathrm{deg}^{-2}$. In the first case, we use the spectroscopic follow-up of X-ray sources by Barger et al. (2003), which is $87 \%$ complete for sources with $R<24$ mag. Here, our classification scheme was applied directly to their data, finding that a low number of unobscured AGN was found, which can be explained by the optical nature of the sources selected for spectroscopic follow-up. Also, a larger number of galaxies relative to other surveys can be seen. This can be explained by the very deep X-ray coverage in the CDF-N, which allows for the detection of a large number of sources with low $f_{X} / f_{\text {opt }}$ and high spatial density, like non-active galaxies.

In the CDF-S, our results were compared with the spectroscopic identifications of Xray sources from Szokoly et al. (2004). In this case, spectra were obtained for 168 X-ray sources and identifications are $60 \%$ complete for sources with $R<24$ mag. Compared to the CYDER survey, the source composition is similar, even though a larger number of Xray normal galaxies is found in the CDF-S, as expected given its fainter X-ray sensitivity. However, the fractions of obscured to unobscured AGN are similar (within $\sim 10 \%$ ) which can be explained by the similarities in the spectroscopic follow-up programs, since both CYDER and CDF-S are $\sim 50 \%$ complete for X-ray sources with $R<24$ mag.

\section{Discussion}

The CYDER survey is located in an intermediate regime in terms of area coverage and sensitivity. A critical step in understanding the properties of the X-ray population is the existence of extensive follow-up at other wavelengths. In particular, optical spectroscopy plays a key role, allowing us to determine redshifts and to identify the origin of the X-ray emission. Therefore, most X-ray surveys are limited by their ability to obtain spectroscopic identifications for a large fraction of the sources, hopefully without biasing the sample. In the case of the CYDER survey, we used $8 \mathrm{~m}$ class telescopes in order to extend the spectroscopic 
coverage to fainter optical magnitudes, namely to $R \simeq 24$ mag.

From Table 5, it is clear that the kind of X-ray sources identified in surveys depends directly on the depth of the optical spectroscopy follow-up. For example, unobscured AGN are bright in the optical bands, therefore in surveys with shallow optical follow-up mostly unobscured AGN are detected (e.g., ChaMP). On the other hand, deep X-ray coverage, together with an extensive spectroscopy campaign based mostly on the Keck 10-m telescopes, allows the CDF-N to detect more faint optical counterparts. Therefore, the population in very deep surveys is dominated by normal galaxies to the CDF-N depths and obscured AGN in the CDF-S range.

In our survey, a total of $50(47.2 \%)$ broad-line AGN were detected. While all of them have a hard X-ray luminosity $L_{X}>10^{42} \mathrm{ergs} \mathrm{s}^{-1}$, two thirds of them have $L_{X}>10^{44} \mathrm{ergs} \mathrm{s}^{-1}$ and therefore are classified as quasars. The average redshift for the broad line sample is $<z>\sim 1.82$, which is much higher than the value found for the remaining X-ray sources. This is clearly explained by the greater optical brightness of unobscured AGN relative to other X-ray emitters.

Using a combination of HR and X-ray luminosity together with optical spectroscopy is very useful for classifying X-ray sources (Szokoly et al. 2004). In Figure 15, the HR versus hard X-ray luminosity diagram is presented. In this case we used a $\mathrm{HR}=-0.2$ in $\mathrm{AGN}$ dominated sources rather than the optical spectra to separate obscured and unobscured AGN, which is equivalent to an effective column density $N_{H} \simeq 4 \times 10^{21} \mathrm{~cm}^{-2}$ for spectral index $\Gamma=1.9$ or $N_{H} \simeq 3 \times 10^{21} \mathrm{~cm}^{-2}$ for $\Gamma=1.7$, so this is a conservative cut to the number of obscured AGN. Also, quasar-like sources are distinguished from other X-ray sources using $L_{X}>10^{44} \mathrm{ergs} \mathrm{s}^{-1}$ as a dividing line. Except for one source described in $\S 4.4$, all the quasars have broad emission lines in their optical spectrum. Most sources that show broad emission lines have $\mathrm{HR}<-0.2$, meaning that they have little or no absorption in X-rays, consistent with their unabsorbed AGN optical spectrum. For non-AGN dominated X-ray emission, no correlation is found between $\mathrm{HR}$ and X-ray luminosity. Also, these sources do not have a characteristic HR value and very hard or soft sources can be found. This X-ray emission is expected to be mostly from high-mass X-ray binaries and type-II supernova remnants in spiral galaxies while for elliptical galaxies the X-ray emission is most likely dominated by hot gas with some contribution from low mass X-ray binaries. Therefore, given the wide range of different X-ray emitter classes together with the lower luminosity, which leads to lower fluxes and therefore larger errors in the HR measurements, can explain why there is no clear correlation between HR and X-ray luminosity and there is no characteristic HR value for low-luminosity, non-AGN X-ray emitters.

For AGN-dominated sources, the relation between the $f_{X} / f_{\text {opt }}$ ratio and Hard X-ray 
luminosity $\left(L_{X}\right)$ is investigated in Figure 16. For sources classified optically as unobscured AGN there is no correlation between $f_{X} / f_{\text {opt }}$ and X-ray luminosity, while for obscured AGN there is a clear correlation in the sense that obscured sources with lower X-ray luminosity have lower $f_{X} / f_{\text {opt }}$ while the hard X-ray sources with large X-ray luminosity also have systematically larger values of $f_{X} / f_{\text {opt }}$. This effect can be explained if the optical light detected in obscured AGN is dominated by the emission from the host galaxy (e.g., Treister et al. 2004b), that is nearly independent from the AGN luminosity. Therefore, for obscured sources that are luminous in X-rays, we can expect a larger $f_{X} / f_{\text {opt }}$ ratio, as observed in our sample. Performing a linear fit to the observed sample of sources optically classified as obscured AGN we obtain a correlation at $\sim 2 \sigma$ significance using the minimum $\chi^{2}$ test, with best-fit parameters given by

$$
\log L_{X}=-39.79( \pm 4.04)+0.917( \pm 0.094) \log \left(f_{X} / f_{\text {opt }}\right)
$$

This correlation is shown by the solid line in Figure 16. This trend can also be observed at the same significance level if the $I$-band optical flux is used instead. This can be explained since the $V$ band is bluer and therefore it is more affected by dust obscuration, while in the $I$ band the host galaxy is more luminous, and therefore in both cases the host galaxy emission dominates over the AGN optical radiation. A similar relation between $f_{X} / f_{\text {opt }}$ and X-ray luminosity for obscured AGN was found by Fiore et al. (2003) in the High Energy Large Area Survey (HELLAS2XMM). Even though they used the $R$ band to calculate the optical luminosity, the correlations are similar.

Given the difficulties in finding obscured AGN at $z>1$, we are not able to disentangle a dependence of the obscured to unobscured AGN numbers ratio with redshift from the strong selection effects on the sample. However, from Figure 13 there is some indication that this ratio can depend on the observed X-ray luminosity. In order to investigate this effect in more detail, in Figure 17 the fraction of obscured to all AGN is shown as a function of hard X-ray luminosity combining the hard X-ray sources detected in the CYDER survey with 77 AGN with $L_{X}>10^{42} \mathrm{ergs} \mathrm{s}^{-1}$ located in the GOODS-S field with identifications and redshifts reported by Szokoly et al. (2004) in order to increase the number of X-ray sources in each bin. This figure clearly shows that a dependence of the fraction of obscured AGN with X-ray luminosity can be observed. A similar trend was first observed by Lawrence \& Elvis (1982) and is consistent with the relation reported by Ueda et al. (2003) and Hasinger (2004).

In other to further investigate this observed correlation, and to determine if it can be explained by selection effects, we used the AGN population models of Treister et al. (2004b). Originally used to predict the AGN number counts in any wavelength from far infrared to Xrays, the Treister et al. (2004b) model is based on the Ueda et al. (2003) luminosity function and its luminosity-dependent density evolution in which the intrinsic $N_{H}$ distribution comes 
from a very simple unified model in which the intrinsic obscured to unobscured AGN ratio is set to be 3:1. The AGN spectral energy distribution is modeled based on three parameters, namely the intrinsic X-ray luminosity of the central engine, the neutral hydrogen column density in the line-of-sight and the redshift of the source in order to compare fluxes in one wavelength to another. Even though this model was applied to the GOODS survey, it can be applied to any other X-ray survey if the proper flux limit and area coverage are used. Given that the luminosity function and AGN SED library in this model are fixed, there is no free parameter to adjust.

In Figure 17 we show the predicted correlation between the fraction of obscured to all AGN and hard X-ray luminosity for sources with $R \lesssim 24$ mag (i.e., the optical flux limit for spectroscopy) both for intrinsic (dot-dashed line) and observed (i.e., adding the effects of obscuration and K-correction; solid line) X-ray luminosity. In both cases, a decrease in the fraction of obscured to all AGN is observed with increasing luminosity, even when the intrinsic ratio is fixed and set to 3:4. Therefore, this observed correlation can be explained as a selection effect since for obscured AGN their lower optical flux makes them harder to detect in spectroscopic surveys, in particular at higher redshifts where most of the more luminous AGN are located.

A crude way to estimate the intrinsic neutral hydrogen column density $\left(N_{H}\right)$ in the line of sight is based on the measured HR. In order to estimate $N_{H}$, we assumed that the intrinsic X-ray spectrum of an AGN can be described by a power law with photon index $\Gamma=1.9$ (e.g., Nandra \& Pounds 1994; Nandra et al. 1997; Mainieri et al. 2002). We then generated a conversion table using XSPEC (Arnaud 1996) to calculate the expected HR for $N_{H}$ in the range $10^{20}-10^{24} \mathrm{~cm}^{-2}$ and redshifts from $z=0$ to $z=5$. The spectral response of the ACIS camera was considered in this calculation. Also, the amount of Galactic absorption in each field as calculated based on the observations by Stark et al. (1992) was added, since all the X-ray emission from extragalactic sources passes through the intergalactic medium of our galaxy. Then, using this conversion table, the observed HR can be translated into a $N_{H}$ value, taking into account the redshift of the source. Even though for individual sources this method to estimate $N_{H}$ may not be very accurate, given the uncertainties associated to perform spectral fitting based on only two bins, these individual uncertainties average out in the distribution.

Given that the ACIS camera is more sensitive in the soft X-ray band, we decided to exclude sources not detected in the hard band, in order to use only the sources for which the HR can give a reasonable idea of the X-ray spectrum. For AGN-dominated sources (i.e. $L_{X}>10^{42} \mathrm{ergs} \mathrm{s}^{-1}$ ), this choice eliminates $34 \%$ of the sources. By cutting the sample to the sources detected in the hard band, a similar fraction of objects optically classified 
as obscured and unobscured AGN are removed from the sample and therefore we do not expect a significant bias introduced by this choice that on the other hand, allows a more precise statistical analysis, since a definite flux limit can be used. Also, sources dominated by AGN emission in X-ray have hard spectrum so if only sources detected in the hard band are considered, the contamination by non-AGN X-ray emitters is reduced. Therefore even sources detected with high significance in the soft band and not detected in the hard band are removed from the following analysis.

The $N_{H}$ distribution for the sources in the reduced sample is presented in Figure 18. While a significant number of sources, $23 \%$, have $N_{H}$ values consistent with no absorption (plotted at $N_{H}=10^{20} \mathrm{~cm}^{-2}$ ), some sources present moderate to high levels of absorption, with $N_{H}>10^{23} \mathrm{~cm}^{-2}(\sim 12 \%)$. The $N_{H}$ distribution for the X-ray sources in the GOODS survey (Dickinson \& Giavalisco 2002; Giavalisco et al. 2004), which overlaps with the Chandra Deep Fields North and South, was calculated previously following a similar procedure by Treister et al. (2004b). The results of this calculation are also presented in Figure 18 scaled to the number of sources in the CYDER survey. Comparing the results from these two surveys with the predictions for the intrinsic $N_{H}$ distribution based on a simple AGN unified model and the Ueda et al. (2003) luminosity function made by Treister et al. (2004b), we found that the obscuration bias is more important for CYDER than for GOODS, meaning that sources with $N_{H}>3 \times 10^{22} \mathrm{~cm}^{-2}$ are preferentially missed in the CYDER survey, since obscuration makes them fainter even in the hard X-ray bands.

Using the AGN number counts predictions by Treister et al. (2004b) adapted to the CYDER flux limits and area coverage, the observed hard X-ray flux distribution is compared to the predictions by this model (Figure 19). When this sample is compared to the predictions by the Treister et al. (2004b) model the results are very encouraging, showing a very good agreement characterized by a K-S confidence level to accept the null hypothesis of $\sim 96 \%$. Using this model, in Figure 19 the predicted contribution by unobscured (type 1; dashed line) and obscured (type 2; dotted line) AGN are shown.

While in the Treister et al. (2004b) model the intrinsic ratio of obscured to unobscured AGN is 3:1 (using $N_{H}=10^{22} \mathrm{~cm}^{-2}$ as the dividing point), the prediction for the CYDER $\mathrm{X}$-ray sample is a ratio of 2.35:1 when the survey flux limit in the X-ray bands is considered. This is consistent with the claim that sources with $N_{H}>3 \times 10^{22} \mathrm{~cm}^{-2}$ are preferentially missed in the CYDER X-ray sample. However, this ratio should be compared to the value of 0.76:1 obtained previously using optical spectroscopy to separate obscured and unobscured AGN. This significant reduction in the relative number of sources classified as obscured AGN can be explained by the optical magnitude cut introduced when optical spectroscopy is used. In the case of the CYDER multiwavelength follow-up, only sources with $V<25$ mag have 
optical spectroscopy, and the completeness level decreases strongly with decreasing optical flux (Figure 4). Since obscured AGN are in general faint optical sources (e.g. Alexander et al. 2001; Koekemoer et al. 2002; Treister et al. 2004b), they are harder to identify using spectroscopy, which cause their relative number to decrease when compared to other X-ray sources that are brighter in the optical bands, like unobscured AGN.

\section{Conclusions}

We presented here the first results from the multiwavelength study of the X-ray sources in the CYDER survey. In this work, we studied the optical and X-ray properties of 267 sources detected in 5 fields observed by Chandra and available in the archive, covering a total of $\sim 0.1 \mathrm{deg}^{-2}$ and spanning a flux range of $10^{-15}-10^{-13} \mathrm{ergs} \mathrm{cm}^{-2} \mathrm{~s}^{-1}$.

The X-ray flux distribution of CYDER sources follows a $\log N-\log S$ relation, both cumulative and differential, that is consistent with the observations in existing X-ray survey. The cumulative $\log N-\log S$ distribution is consistent with the observations of Ueda et al. (2003), while the differential $\log N-\log S$ is in good agreement with the distribution derived by the SEXSI survey (Harrison et al. 2003). This implies that there are not significant variations in this sample compared to other existing surveys, and therefore that the results can be directly compared.

In general, sources optically classified as obscured AGN have redder optical colors than unobscured AGN and are closer to the colors of normal galaxies, as expected from the unification model of AGN. Also, a correlation between $f_{X} / f_{\text {opt }}$ and hard X-ray luminosity is observed in the sample of sources optically classified as obscured AGN.

The ratio of obscured AGN seems to be changing as a function of X-ray luminosity, in the sense that for more luminous sources the ratio of obscured to unobscured AGN is lower than for less luminous objects. However, this relation can be explained as a selection effect since obscured AGN are fainter in the optical bands and therefore harder to identify for spectroscopic surveys. In fact, the observed correlation can be reproduced using the Treister et al. (2004b) models that have a fixed intrinsic ratio of 3:4 if an optical cut of $R \lesssim 24 \mathrm{mag}$ (i.e., the magnitude limit for spectroscopy) is used.

The $N_{H}$ distribution for sources in the CYDER survey is consistent with the predicted distribution by Treister et al. (2004b) assuming a torus geometry for the obscuring material once selection effects are accounted. This implies that X-ray surveys are subject to significant incompleteness for sources with large amounts of absorption. In the particular case of the CYDER survey this incompleteness is important for sources with $N_{H}>3 \times 10^{22} \mathrm{~cm}^{-2}$. How- 
ever, once these selection effects are accounted for, the observed hard X-ray flux distribution is consistent with the predictions of the models of Treister et al. (2004b).

ET would like to thank the support of Fundación Andes, Centro de Astrofisica FONDAP and the Sigma-Xi foundation through a Grant in-aid of Research. This material is based upon work supported by the National Science Foundation under Grant No. AST-0201667, an Astronomy \& Astrophysics Postdoctoral Fellowship awarded to E. Gawiser. We thank the anonymous referee for a very careful review and a constructive report that improved the presentation of this paper. We would like to thank the help of Steve Zepf, Rafael Guzman and Maria Teresa Ruiz in the original design of this survey. We also thank the assistance during the observations provided by the staff at Las Campanas Observatory, Cerro Tololo International Observatory and Cerro Paranal.

\section{REFERENCES}

Akiyama, M., Ohta, K., Yamada, T., Kashikawa, N., Yagi, M., Kawasaki, W., Sakano, M., Tsuru, T., Ueda, Y., Takahashi, T., Lehmann, I., Hasinger, G., \& Voges, W. 2000, ApJ, 532, 700

Alexander, D. M., Bauer, F. E., Brandt, W. N., Schneider, D. P., Hornschemeier, A. E., Vignali, C., Barger, A. J., Broos, P. S., Cowie, L. L., Garmire, G. P., Townsley, L. K., Bautz, M. W., Chartas, G., \& Sargent, W. L. W. 2003, AJ, 126, 539

Alexander, D. M., Brandt, W. N., Hornschemeier, A. E., Garmire, G. P., Schneider, D. P., Bauer, F. E., \& Griffiths, R. E. 2001, AJ, 122, 2156

Arnaud, K. A. 1996, in ASP Conf. Ser. 101: Astronomical Data Analysis Software and Systems V, 17-+

Barger, A. J., Cowie, L. L., Capak, P., Alexander, D. M., Bauer, F. E., Fernandez, E., Brandt, W. N., Garmire, G. P., \& Hornschemeier, A. E. 2003, AJ, 126, 632

Bertin, E. \& Arnouts, S. 1996, A\&AS, 117, 393

Brandt, W. N., Alexander, D. M., Hornschemeier, A. E., Garmire, G. P., Schneider, D. P., Barger, A. J., Bauer, F. E., Broos, P. S., Cowie, L. L., Townsley, L. K., Burrows, D. N., Chartas, G., Feigelson, E. D., Griffiths, R. E., Nousek, J. A., \& Sargent, W. L. W. 2001, AJ, 122, 2810 
Castander, F. J., Treister, E., Maccarone, T. J., Coppi, P. S., Maza, J., Zepf, S. E., \& Guzmán, R. 2003a, AJ, 125, 1689

Castander, F. J., Treister, E., Maza, J., Coppi, P. S., Maccarone, T. J., Zepf, S. E., Guzmán, R., \& Ruiz, M. T. 2003b, Astronomische Nachrichten, 324, 40

Ciliegi, P., Elvis, M., Wilkes, B. J., Boyle, B. J., \& McMahon, R. G. 1997, MNRAS, 284, 401

Comastri, A., Setti, G., Zamorani, G., \& Hasinger, G. 1995, A\&A, 296, 1

Dickinson, M. \& Giavalisco, M. 2002, in The Mass of Galaxies at Low and High Redshift, in press, astro-ph/0204213

Fioc, M. \& Rocca-Volmerange, B. 1997, A\&A, 326, 950

Fiore, F., Brusa, M., Cocchia, F., Baldi, A., Carangelo, N., Ciliegi, P., Comastri, A., La Franca, F., Maiolino, R., Matt, G., Molendi, S., Mignoli, M., Perola, G. C., Severgnini, P., \& Vignali, C. 2003, A\&A, 409, 79

Gandhi, P., Crawford, C. S., Fabian, A. C., \& Johnstone, R. M. 2004, MNRAS, 348, 529

Garmire, G. P., Bautz, M. W., Ford, P. G., Nousek, J. A., \& Ricker, G. R. 2003, in X-Ray and Gamma-Ray Telescopes and Instruments for Astronomy. Edited by Joachim E. Truemper, Harvey D. Tananbaum. Proceedings of the SPIE, Volume 4851, pp. 28-44 (2003)., 28-44

Giacconi, R., et al. 2001, ApJ, 551, 624

Giacconi, R., Zirm, A., Wang, J., Rosati, P., Nonino, M., Tozzi, P., Gilli, R., Mainieri, V., Hasinger, G., Kewley, L., Bergeron, J., Borgani, S., Gilmozzi, R., Grogin, N., Koekemoer, A., Schreier, E., Zheng, W., \& Norman, C. 2002, ApJS, 139, 369

Giavalisco, M. et al. 2004, ApJ, 600, L93

Gilli, R., Cimatti, A., Daddi, E., Hasinger, G., Rosati, P., Szokoly, G., Tozzi, P., Bergeron, J., Borgani, S., Giacconi, R., Kewley, L., Mainieri, V., Mignoli, M., Nonino, M., Norman, C., Wang, J., Zamorani, G., Zheng, W., \& Zirm, A. 2003, ApJ, 592, 721

Gilli, R., Risaliti, G., \& Salvati, M. 1999, A\&A, 347, 424

Gilli, R., Salvati, M., \& Hasinger, G. 2001, A\&A, 366, 407 
Gioia, I. M., Maccacaro, T., Schild, R. E., Wolter, A., Stocke, J. T., Morris, S. L., \& Henry, J. P. 1990, ApJS, 72, 567

Green, P. J., Silverman, J. D., Cameron, R. A., Kim, D.-W., Wilkes, B. J., Barkhouse, W. A., LaCluyzé, A., Morris, D., Mossman, A., Ghosh, H., Grimes, J. P., Jannuzi, B. T., Tananbaum, H., Aldcroft, T. L., Baldwin, J. A., Chaffee, F. H., Dey, A., Dosaj, A., Evans, N. R., Fan, X., Foltz, C., Gaetz, T., Hooper, E. J., Kashyap, V. L., Mathur, S., McGarry, M. B., Romero-Colmenero, E., Smith, M. G., Smith, P. S., Smith, R. C., Torres, G., Vikhlinin, A., \& Wik, D. R. 2004, ApJS, 150, 43

Gruber, D. E., Matteson, J. L., Peterson, L. E., \& Jung, G. V. 1999, ApJ, 520, 124

Harrison, F. A., Eckart, M. E., Mao, P. H., Helfand, D. J., \& Stern, D. 2003, ApJ, 596, 944

Hasinger, G. 2004, Nucl. Phys. Proc. Suppl., 132, 86

Hasinger, G., Burg, R., Giacconi, R., Schmidt, M., Trumper, J., \& Zamorani, G. 1998, A\&A, 329,482

Ho, L. C. 1999, ApJ, 516, 672

Hornschemeier, A. E., et al. 2003, AJ, 126, 575

Kim, D.-W., Cameron, R. A., Drake, J. J., Evans, N. R., Freeman, P., Gaetz, T. J., Ghosh, H., Green, P. J., Harnden, F. R., Karovska, M., Kashyap, V., Maksym, P. W., Ratzlaff, P. W., Schlegel, E. M., Silverman, J. D., Tananbaum, H. D., Vikhlinin, A. A., Wilkes, B. J., \& Grimes, J. P. 2004, ApJS, 150, 19

Koekemoer, A. M., Grogin, N. A., Schreier, E. J., Giacconi, R., Gilli, R., Kewley, L., Norman, C., Zirm, A., Bergeron, J., Rosati, P., Hasinger, G., Tozzi, P., \& Marconi, A. 2002, ApJ, 567, 657

Landolt, A. U. 1992, AJ, 104, 340

Lawrence, A. \& Elvis, M. 1982, ApJ, 256, 410

Lira, P., Ward, M., Zezas, A., Alonso-Herrero, A., \& Ueno, S. 2002, MNRAS, 330, 259

Madau, P., Ghisellini, G., \& Fabian, A. C. 1994, MNRAS, 270, L17+

Mainieri, V., Bergeron, J., Hasinger, G., Lehmann, I., Rosati, P., Schmidt, M., Szokoly, G., \& Della Ceca, R. 2002, A\&A, 393, 425

Moretti, A., Campana, S., Lazzati, D., \& Tagliaferri, G. 2003, ApJ, 588, 696 
Morrison, R. \& McCammon, D. 1983, ApJ, 270, 119

Mukai, K. 1993, Legacy, vol. 3, p.21-31, 3, 21

Muller, G. P., Reed, R., Armandroff, T., Boroson, T. A., \& Jacoby, G. H. 1998, in Proc. SPIE Vol. 3355, p. 577-585, Optical Astronomical Instrumentation, Sandro D’Odorico; Ed., $577-585$

Mushotzky, R. F., Cowie, L. L., Barger, A. J., \& Arnaud, K. A. 2000, Nature, 404, 459

Nandra, K., George, I. M., Mushotzky, R. F., Turner, T. J., \& Yaqoob, T. 1997, ApJ, 476, 70

Nandra, K. \& Pounds, K. A. 1994, MNRAS, 268, 405

O’Sullivan, E., Forbes, D. A., \& Ponman, T. J. 2001, MNRAS, 328, 461

Rosati, P., Tozzi, P., Giacconi, R., Gilli, R., Hasinger, G., Kewley, L., Mainieri, V., Nonino, M., Norman, C., Szokoly, G., Wang, J. X., Zirm, A., Bergeron, J., Borgani, S., Gilmozzi, R., Grogin, N., Koekemoer, A., Schreier, E., \& Zheng, W. 2002, ApJ, 566, 667

Spergel, D. N., Verde, L., Peiris, H. V., Komatsu, E., Nolta, M. R., Bennett, C. L., Halpern, M., Hinshaw, G., Jarosik, N., Kogut, A., Limon, M., Meyer, S. S., Page, L., Tucker, G. S., Weiland, J. L., Wollack, E., \& Wright, E. L. 2003, ApJS, 148, 175

Stark, A. A., Gammie, C. F., Wilson, R. W., Bally, J., Linke, R. A., Heiles, C., \& Hurwitz, M. 1992, ApJS, 79, 77

Szokoly, G. et al. 2004, ApJS, in press, astro-ph/0312324

Treister, E., Castander, F. J., Maccarone, T. J., Herrera, D., Gawiser, E., Maza, J., \& Coppi, P. S. 2004a, ApJ, 603, 36

Treister, E. et al. 2004b, ApJ, in press, astro-ph/0408099

Ueda, Y., Akiyama, M., Ohta, K., \& Miyaji, T. 2003, ApJ, 598, 886

Vanden Berk, D. E., et al. 2001, AJ, 122, 549 
Table 1. CYDER Fields

\begin{tabular}{|c|c|c|c|c|c|c|c|c|c|c|}
\hline ID & Obs. ID & Target & $\begin{array}{l}\text { Exposure } \\
\text { Time (ks) }\end{array}$ & Mode & $\begin{array}{c}\mathrm{RA} \\
(\mathrm{J} 2000)\end{array}$ & $\begin{array}{c}\text { Dec } \\
(\mathrm{J} 2000)\end{array}$ & $\begin{array}{l}\text { Gal } \\
\text { Lat. }\end{array}$ & $\begin{array}{c}\text { Gal N }{ }_{H} \\
\left(10^{20} \mathrm{~cm}^{-2}\right)\end{array}$ & $\begin{array}{l}\text { Eff. Area } \\
\left(\operatorname{arcmin}^{2}\right)\end{array}$ & $\begin{array}{c}\text { Hard Flux Lim } \\
\left(\mathrm{erg} \mathrm{cm}^{-2} \mathrm{~s}^{-1}\right)\end{array}$ \\
\hline $\mathrm{C} 2$ & 921 & HCG 62 & 49.15 & ACIS-S & $12^{\mathrm{h}} \cdot 53^{\mathrm{m}} 05^{\mathrm{s}} \cdot 7$ & $-09.12 \cdot 20^{\prime \prime} 0$ & $53.66^{\circ}$ & 3.11 & 124.0 & $7.9 \times 10^{-15}$ \\
\hline C5 & 866 & Q1127-145 & 30.16 & ACIS-S & $11^{\mathrm{h}} 30^{\mathrm{m}} 07^{\mathrm{s}} \cdot 1$ & $-14.49^{\prime} \cdot 27^{\prime \prime} 0$ & $43.64^{\circ}$ & 4.04 & 0.0 & $1.6 \times 10^{-14}$ \\
\hline D1 & 905 & HCG 90 & 50.16 & ACIS-I & $22.02^{\mathrm{m}} \cdot 04^{\mathrm{s}} \cdot 0$ & $-31.58{ }^{\prime} 30^{\prime \prime} 0$ & $-53.08^{\circ}$ & 1.64 & 114.24 & $1.44 \times 10^{-15}$ \\
\hline D2 & 861 & $\mathrm{Q} 2345+007$ & 75.15 & ACIS-S & $23.48^{\mathrm{m}} \cdot 19.6$ & $00.57 ! 21^{\prime \prime} 0$ & $-58.07^{\circ}$ & 3.77 & 62.0 & $1.33 \times 10^{-15}$ \\
\hline D3 & 796 & SBS 0335-052 & 60.51 & ACIS-I & $03^{\mathrm{h}} \cdot 37^{\mathrm{m}} \cdot 44^{\mathrm{s}} \cdot 0$ & $-05.02 ! 399^{\prime \prime} 0$ & $-44.69^{\circ}$ & 4.91 & 120.0 & $3.59 \times 10^{-15}$ \\
\hline
\end{tabular}


Table 2. Summary of Optical Observations

\begin{tabular}{ccccccc}
\hline \hline Field & Instrument & Date & Filters & $\begin{array}{c}\text { Exposure } \\
\text { Time }\end{array}$ & Seeing & $\begin{array}{c}\text { Lim. } \\
\text { Magnitude }(5 \sigma)\end{array}$ \\
\hline C2 & CTIO/MOSAIC & $03 / 11 / 2000$ & $V$ & $6000 \mathrm{~s}$ & $1.1 ”$ & 26.5 \\
C2 & CTIO/MOSAIC & $03 / 11 / 2000$ & $I$ & $1500 \mathrm{~s}$ & $1.0 ”$ & 24.9 \\
C5 & CTIO/MOSAIC & $03 / 12 / 2000$ & $V$ & $6000 \mathrm{~s}$ & $1.1 ”$ & 26.5 \\
C5 & CTIO/MOSAIC & $03 / 12 / 2000$ & $I$ & $1500 \mathrm{~s}$ & $1.0 "$ & 24.9 \\
D1 & CTIO/MOSAIC & $08 / 22 / 2001$ & $V$ & $6600 \mathrm{~s}$ & $1.0 "$ & 26.7 \\
D1 & CTIO/MOSAIC & $08 / 22 / 2001$ & $I$ & $1800 \mathrm{~s}$ & $0.9 ”$ & 25.1 \\
D2 & CTIO/MOSAIC & $08 / 23 / 2001$ & $V$ & $1500 \mathrm{~s}$ & $1.4 ”$ & 25.5 \\
D2 & CTIO/MOSAIC & $08 / 23 / 2001$ & $I$ & $5100 \mathrm{~s}$ & $2.0 ”$ & 24.8 \\
D3 & CTIO/MOSAIC & $10 / 10 / 2002$ & $V$ & $3000 \mathrm{~s}$ & $1.2 ”$ & 26.1 \\
D3 & CTIO/MOSAIC & $10 / 10 / 2002$ & $I$ & $1200 \mathrm{~s}$ & $1.1 ”$ & 24.6 \\
\hline
\end{tabular}

Table 3. Summary of Spectroscopic Observations

\begin{tabular}{cccccc}
\hline \hline Field & Instrument & Date & Masks & $\begin{array}{c}\text { Exp. Time } \\
\text { per Mask }\end{array}$ & Seeing \\
& & & & $6300 \mathrm{~s}$ & $1.0 "$ \\
C2 & VLT/FORS2 & $02 / 14 / 2002$ & 3 & $8000 \mathrm{~s}$ & $1.2 "$ \\
C5 & VLT/FORS2 & $02 / 15 / 2002$ & 1 & $6300 \mathrm{~s}$ & $0.7 "$ \\
D1 & Baade/LDSS-2 & $10 / 03-04 / 2002$ & 3 & $6900 \mathrm{~s}$ & $1.0 "$ \\
D1 & Baade/LDSS-2 & $10 / 03-04 / 2002$ & 3 & $7200 \mathrm{~s}$ & $0.9 "$ \\
D2 & Baade/LDSS-2 & $10 / 03-04 / 2002$ & 2 & $9200 \mathrm{~s}$ & $0.5 "$ \\
D3 & VLT/FORS2 & $10 / 29-31 / 2003$ & 3 & $9200 \mathrm{~s}$ & $0.6 "$ \\
D3 & VLT/FORS2 & $10 / 29-31 / 2003$ & 6 & & \\
\hline
\end{tabular}


Table 4. Catalog of X-ray sources in the CYDER fields.

\begin{tabular}{|c|c|c|c|c|c|c|c|c|c|c|c|c|c|c|c|}
\hline $\begin{array}{l}\text { Name } \\
\text { CXOCY- }\end{array}$ & RA & Dec & Offset & $\begin{array}{c}\text { SB } \\
\text { Counts }\end{array}$ & $\begin{array}{c}\text { HB } \\
\text { Counts }\end{array}$ & $\begin{array}{l}\text { SB Error } \\
\text { Counts }\end{array}$ & $\begin{array}{c}\text { HB Error } \\
\text { Counts }\end{array}$ & $\mathrm{HR}$ & V Mag. & I Mag. & $\begin{array}{c}\text { SB flux } \\
\operatorname{erg~} \mathrm{cm}^{-2} \mathrm{~s}^{-1}\end{array}$ & $\begin{array}{c}\text { HB flux } \\
\text { erg } \mathrm{cm}^{-2} \mathrm{~s}^{-1}\end{array}$ & $\mathrm{z}$ & $\begin{array}{l}\text { SB Lum. } \\
\operatorname{erg~s}^{-1}\end{array}$ & $\begin{array}{l}\mathrm{HB} \mathrm{L} \\
\text { erg s }\end{array}$ \\
\hline J125302.4-091312 & 125302.40 & -09 1311.8 & 1.104 & 380.3 & 184.9 & 20.1 & 13.8 & -0.35 & 20.50 & 20.46 & $3.80 \mathrm{e}-14$ & $7.20 \mathrm{e}-14$ & 1.13 & $2.62 \mathrm{e}+44$ & \\
\hline J125314.8-091301 & 125314.82 & -09 1301.2 & 1.047 & 9.1 & 52.5 & 3.2 & 7.3 & 0.70 & 22.70 & 20.70 & $1.90 \mathrm{e}-15$ & $3.10 \mathrm{e}-14$ & 0.72 & $4.24 \mathrm{e}+42$ & 6.9 \\
\hline J125314.6-091050 & 125314.64 & -091049.8 & - & 24.5 & 8.4 & 5.0 & 3.0 & -0.49 & ¿26.50 & ¿24.90 & $2.60 \mathrm{e}-15$ & $4.80 \mathrm{e}-15$ & - & & \\
\hline J125311.1-091118 & 125311.12 & -09 $11 \quad 17.7$ & 1.027 & 0.0 & 19.8 & 0.0 & 4.6 & 1.00 & 21.34 & 19.96 & $7.00 \mathrm{e}-16$ & $1.60 \mathrm{e}-15$ & 0.48 & $5.84 \mathrm{e}+41$ & 1.33 \\
\hline J125310.4-091024 & 125310.41 & -091023.6 & 0.831 & 36.5 & 20.9 & 6.1 & 4.7 & -0.27 & 22.62 & 21.19 & $4.20 \mathrm{e}-16$ & $8.80 \mathrm{e}-16$ & 0.56 & $5.06 \mathrm{e}+41$ & 1.06 \\
\hline J125306.1-091344 & 125306.08 & -09 1343.9 & - & 28.6 & 32.8 & 6.4 & 6.1 & 0.07 & ¿26.50 & ¿24.90 & $2.50 \mathrm{e}-16$ & $1.10 \mathrm{e}-15$ & - & - & \\
\hline J125306.0-091316 & 125306.00 & -09 1316.4 & 0.912 & 48.8 & 40.7 & 7.7 & 6.8 & -0.09 & 22.60 & 20.06 & $5.70 \mathrm{e}-15$ & $1.70 \mathrm{e}-14$ & 0.72 & $1.28 \mathrm{e}+43$ & \\
\hline J125305.3-090824 & 125305.34 & -090823.6 & 0.592 & 136.2 & 51.6 & 11.7 & 7.3 & -0.45 & 20.58 & 19.70 & $1.50 \mathrm{e}-14$ & $2.10 \mathrm{e}-14$ & 0.50 & $1.40 \mathrm{e}+43$ & \\
\hline J125305.0-091339 & 125305.01 & -09 1339.0 & - & 66.4 & 34.7 & 9.3 & 6.3 & -0.31 & ¿26.50 & ¿24.90 & $7.30 \mathrm{e}-15$ & $1.30 \mathrm{e}-14$ & - & & \\
\hline J125303.8-090810 & 125303.83 & -090809.7 & - & 60.5 & 48.5 & 7.9 & 7.1 & -0.11 & ¿26.50 & ¿24.90 & $8.10 \mathrm{e}-15$ & $2.00 \mathrm{e}-14$ & - & 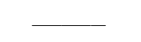 & \\
\hline J125303.0-091242 & 125303.01 & -09 1241.9 & 0.855 & 95.8 & 30.3 & 10.7 & 5.9 & -0.52 & 23.48 & 21.80 & $8.50 \mathrm{e}-15$ & $1.50 \mathrm{e}-14$ & - & & \\
\hline J125302.9-091058 & 125302.93 & -09 1057.7 & 0.651 & 0.0 & 13.8 & 0.0 & 3.9 & 1.00 & 21.77 & 20.56 & $3.60 \mathrm{e}-16$ & $8.40 \mathrm{e}-16$ & 0.38 & $1.76 \mathrm{e}+41$ & 4.11 \\
\hline J125301.9-091134 & 125301.90 & -09 1133.9 & 0.853 & 27.6 & 13.6 & 5.6 & 3.9 & -0.34 & 22.70 & 21.40 & $2.70 \mathrm{e}-15$ & $6.30 \mathrm{e}-15$ & 0.71 & $5.96 \mathrm{e}+42$ & 1.39 \\
\hline J125300.9-090941 & 125300.89 & -090941.3 & 0.904 & 0.0 & 17.7 & 0.0 & 4.4 & 1.00 & 24.85 & 22.55 & $1.10 \mathrm{e}-15$ & $2.40 \mathrm{e}-15$ & $0.97^{\mid}$ & $5.13 \mathrm{e}+42$ & 1.12 \\
\hline J125317.5-091223 & 125317.55 & -09 1223.3 & 0.795 & 23.6 & 9.8 & 5.0 & 3.3 & -0.42 & 26.05 & 24.56 & $2.90 \mathrm{e}-15$ & $4.00 \mathrm{e}-15$ & $-N$ & 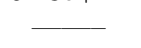 & \\
\hline J125315.2-091424 & 125315.21 & -091424.4 & 1.280 & 0.0 & 52.5 & 0.0 & 7.6 & 1.00 & 24.05 & 22.88 & $1.50 \mathrm{e}-15$ & $2.60 \mathrm{e}-14$ & 1.15 & $1.09 \mathrm{e}+43$ & \\
\hline J125311.8-091339 & 125311.84 & -091338.6 & - & 35.1 & 17.9 & 6.3 & 4.7 & -0.32 & ¿26.50 & ¿24.90 & $3.30 \mathrm{e}-15$ & $8.70 \mathrm{e}-15$ & 3.05 & $2.57 \mathrm{e}+44$ & \\
\hline
\end{tabular}

Note. - This table is published in its entirety in the electronic edition of the Astrophysical Journal. A portion is shown here for guidance regarding its form and content. 
Table 5. Source Compositions in X-ray Surveys

\begin{tabular}{cccccc}
\hline \hline & \multicolumn{5}{c}{ Type } \\
\cline { 2 - 5 } Survey & Stars & Galaxies & Obscured AGN & Unobscured AGN & \\
\hline CYDER & $6.6 \%$ & $10.4 \%$ & $35.8 \%$ & $47.2 \%$ & 1 \\
ChaMP & $9.9 \%$ & $14.1 \%$ & $23.9 \%$ & $52.1 \%$ & 2 \\
CDF-N & $4.9 \%$ & $43.3 \%$ & $39.4 \%$ & $12.4 \%$ & 3 \\
CDF-S & $4.5 \%$ & $17.8 \%$ & $42.7 \%$ & $35.0 \%$ & 4 \\
\hline
\end{tabular}

References. - (1) This paper; (2) Green et al. 2004; (3) Barger et al. 2003; (4) Szokoly et al. 2004 


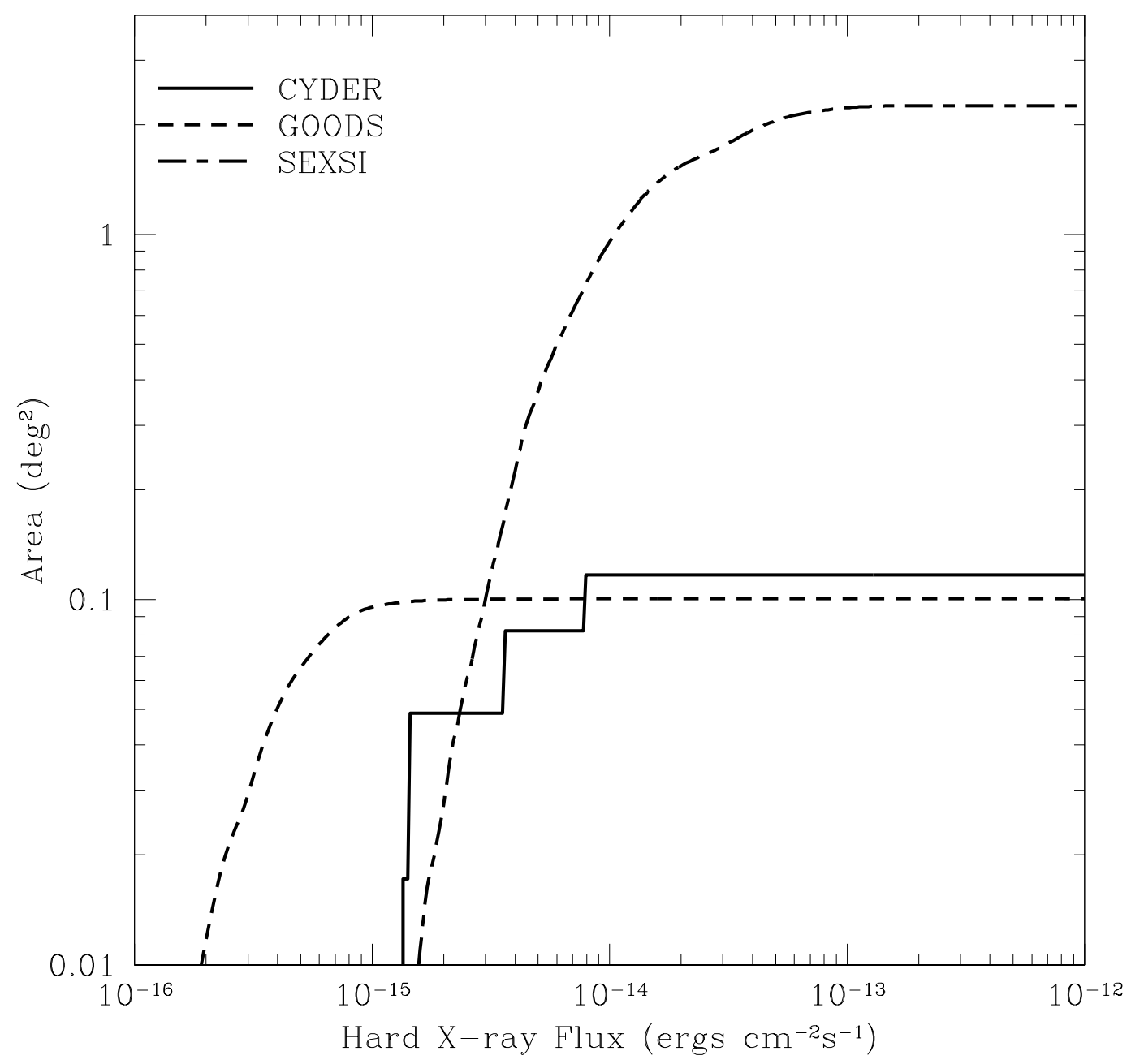

Fig. 1.- Area covered as a function of limiting flux in the hard X-ray band for the CYDER survey, compared to other X-ray surveys like SEXSI (Harrison et al. 2003) and GOODS (Alexander et al. 2003). 


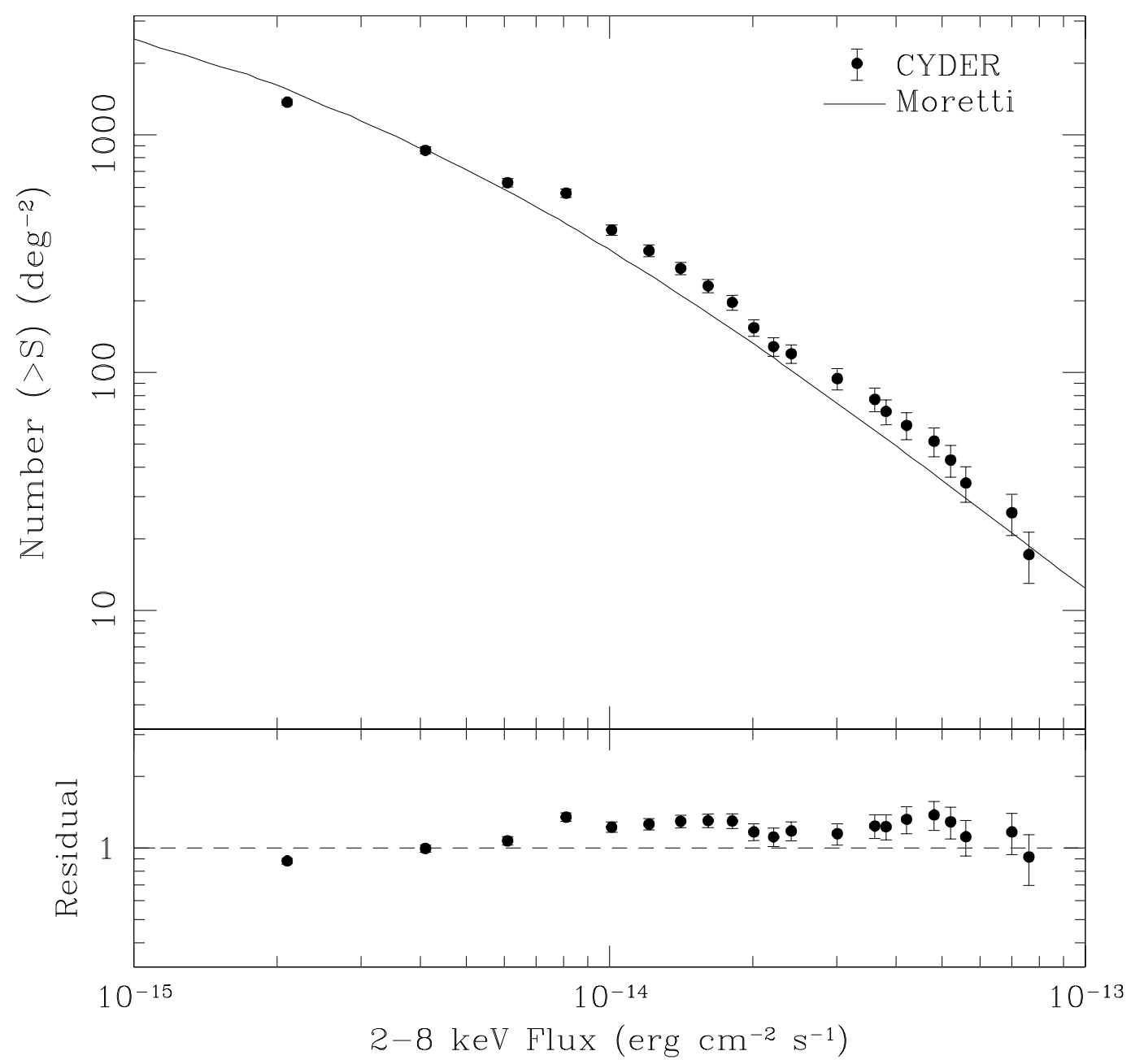

Fig. 2.- Cumulative $\log N-\log S$ plot for sources in the CYDER survey detected in the hard X-ray band. In the upper panel, the solid line shows the relation observed by Moretti et al. (2003) using a combination of shallow and wide and deep pencil beam X-ray surveys, while in the lower panel, residuals calculated as the ratio of observed sources to the numbers obtained by Moretti et al. (2003) are presented. 


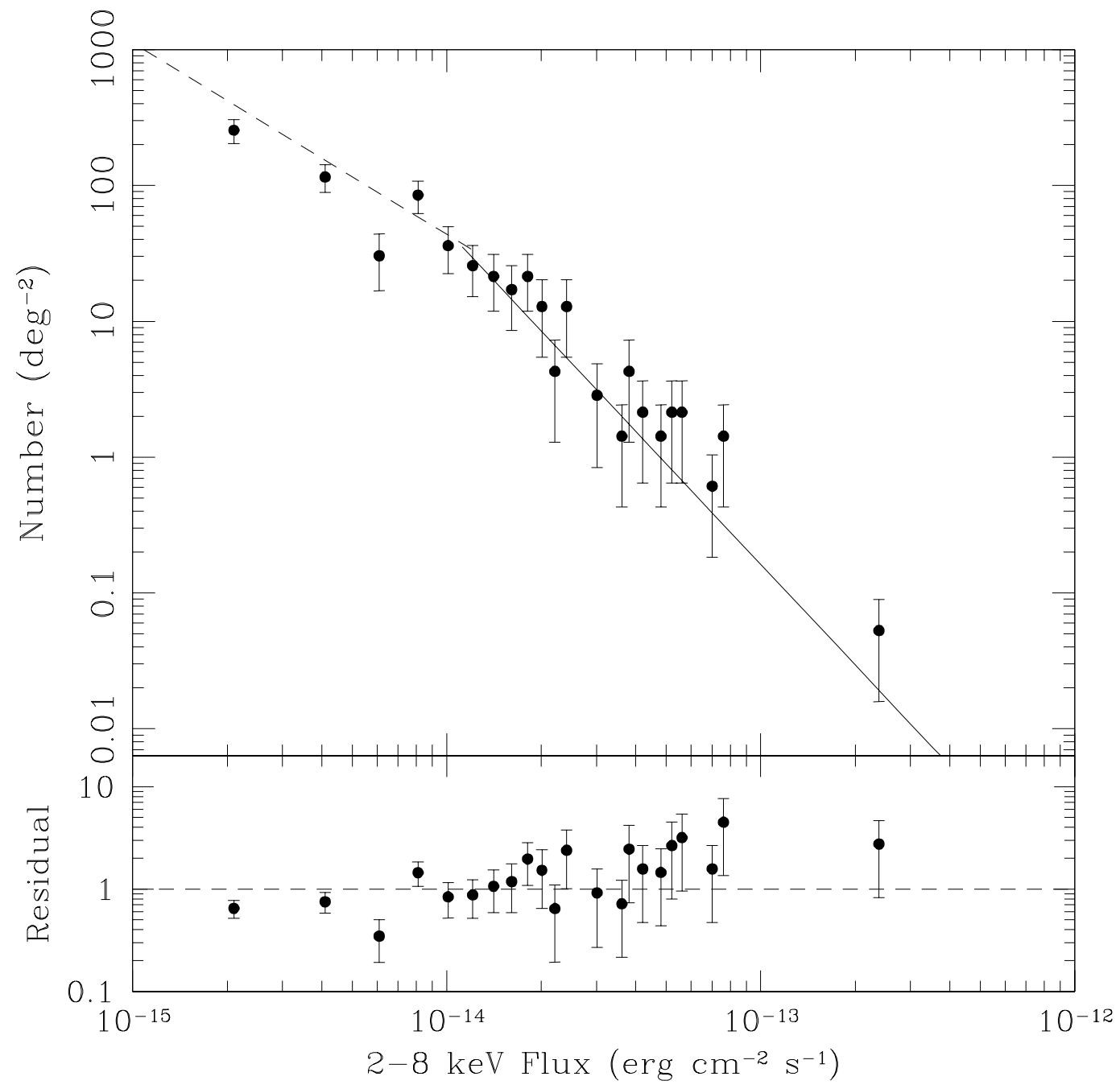

Fig. 3.- Differential $\log N-\log S$ plot. Upper panel shows CYDER data, while the solid line shows the best-fit to the SEXSI counts. In the lower panel, the residuals computed as the ratio between the best-fit curve and the data are shown. Even tough some scatter is present, the fit provides a good description of the distribution of CYDER sources, with a reduced $\chi^{2}$ of 1.37 . 


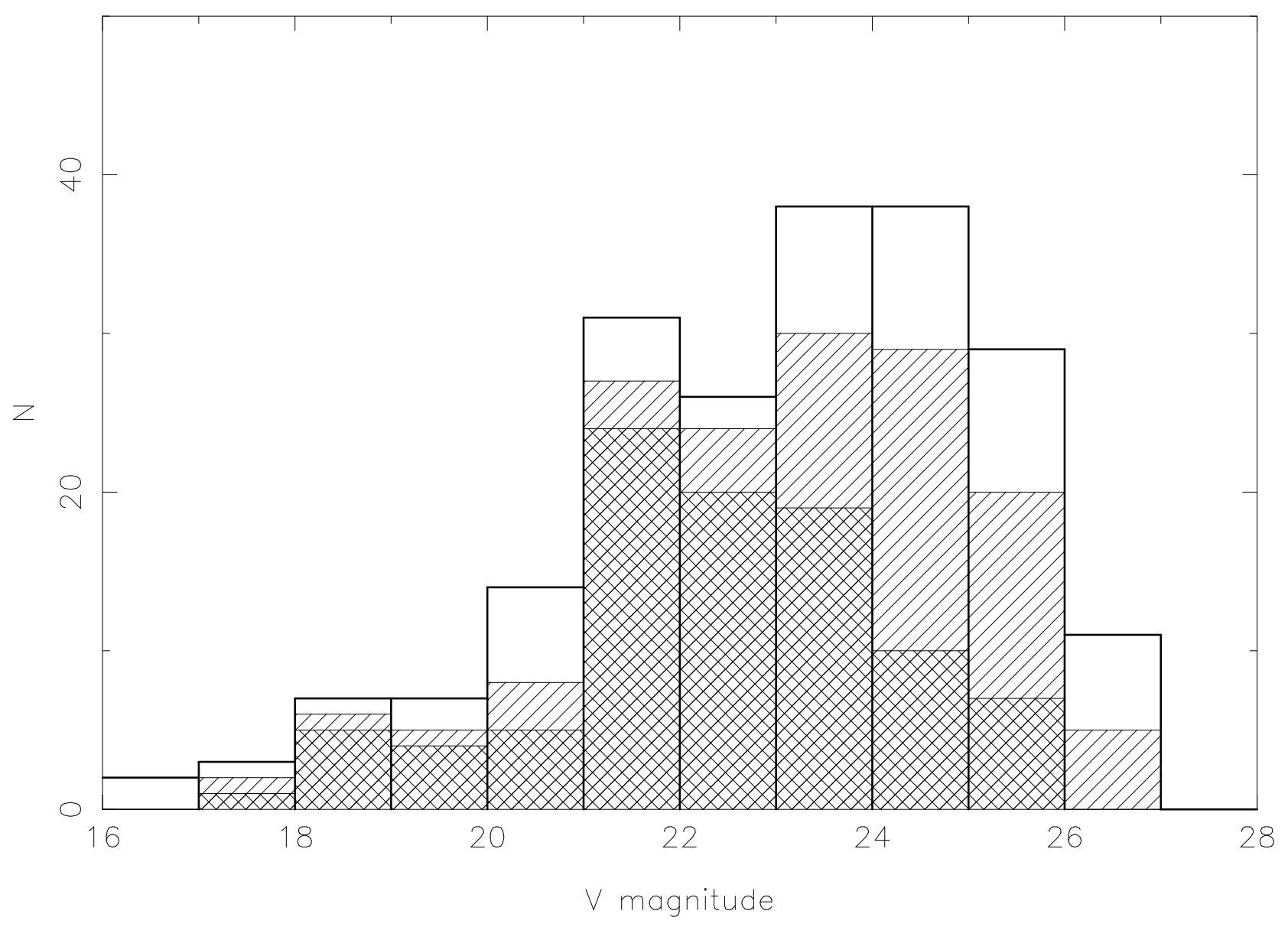

Fig. 4.- $V$ magnitude distribution for X-ray sources with detected optical counterparts in the CYDER fields. Hatched histogram shows the magnitude distribution for sources targeted for optical spectroscopy, while the cross-hatched histogram shows the distribution of sources successfully identified. While sources were selected for spectroscopy independent of their optical properties, it is clear that spectroscopic identifications are much more efficient for $\mathrm{X}$-ray sources brighter in the optical. 


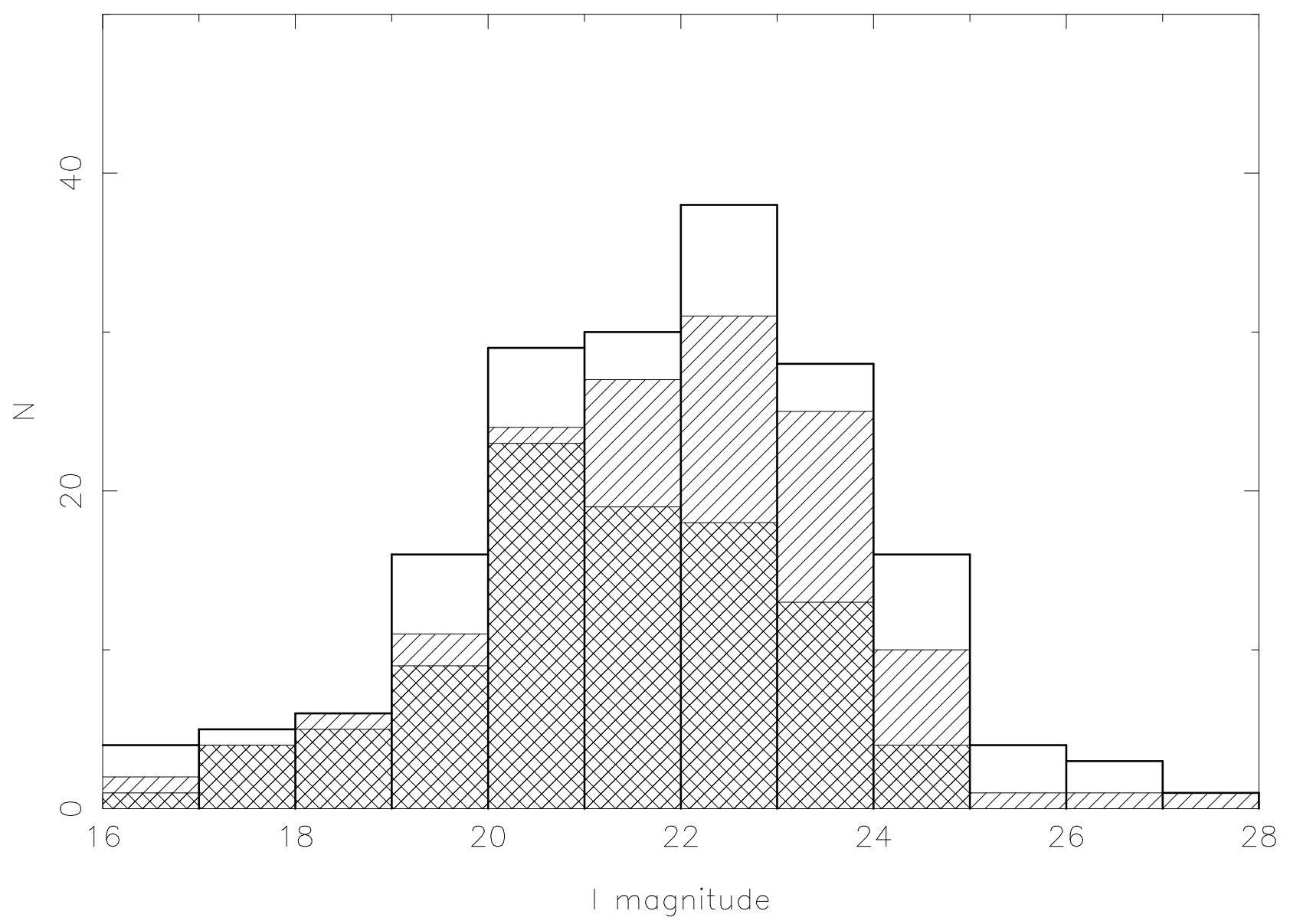

Fig. 5.- I magnitude distribution for X-ray sources with detected $I$-band counterparts. The magnitude distribution for sources targeted for spectroscopy is shown by the hatched histogram, while the distribution for sources successfully identified is shown by the crosshatched histogram. Again, the efficiency of spectroscopic identifications is much higher for sources brighter in the optical bands. 


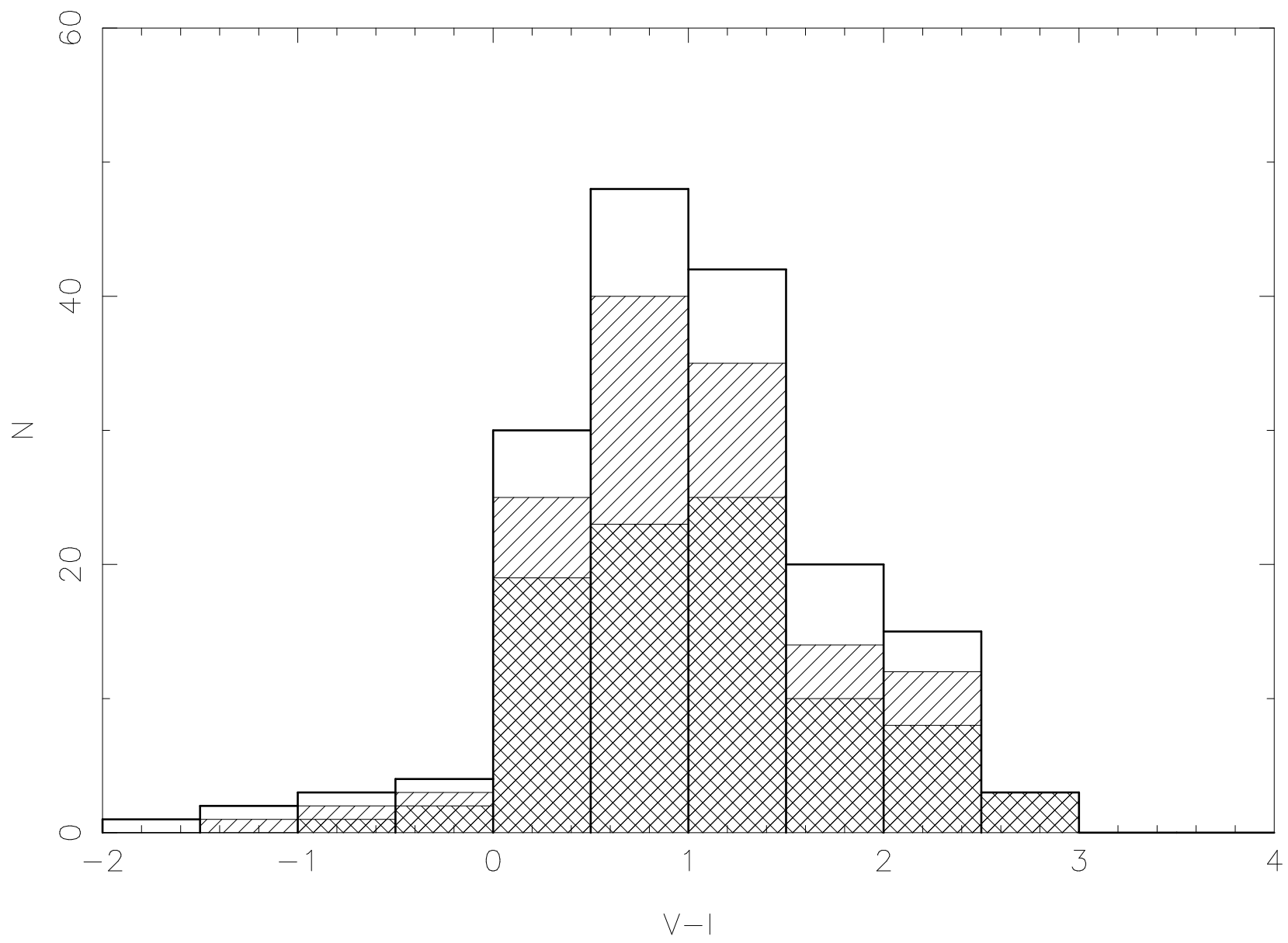

Fig. 6. $-V-I$ color distribution for X-ray sources detected in the optical bands. Hatched histogram shows the distribution for sources targeted for spectroscopy while the cross-hatched histograms shows the distribution for sources successfully identified. In this case, these distributions are very similar, with a K-S confidence level for the null hypothesis of $99.89 \%$, showing that the efficiency of spectroscopic identifications is independent of the $V-I$ color of the optical counterpart. 


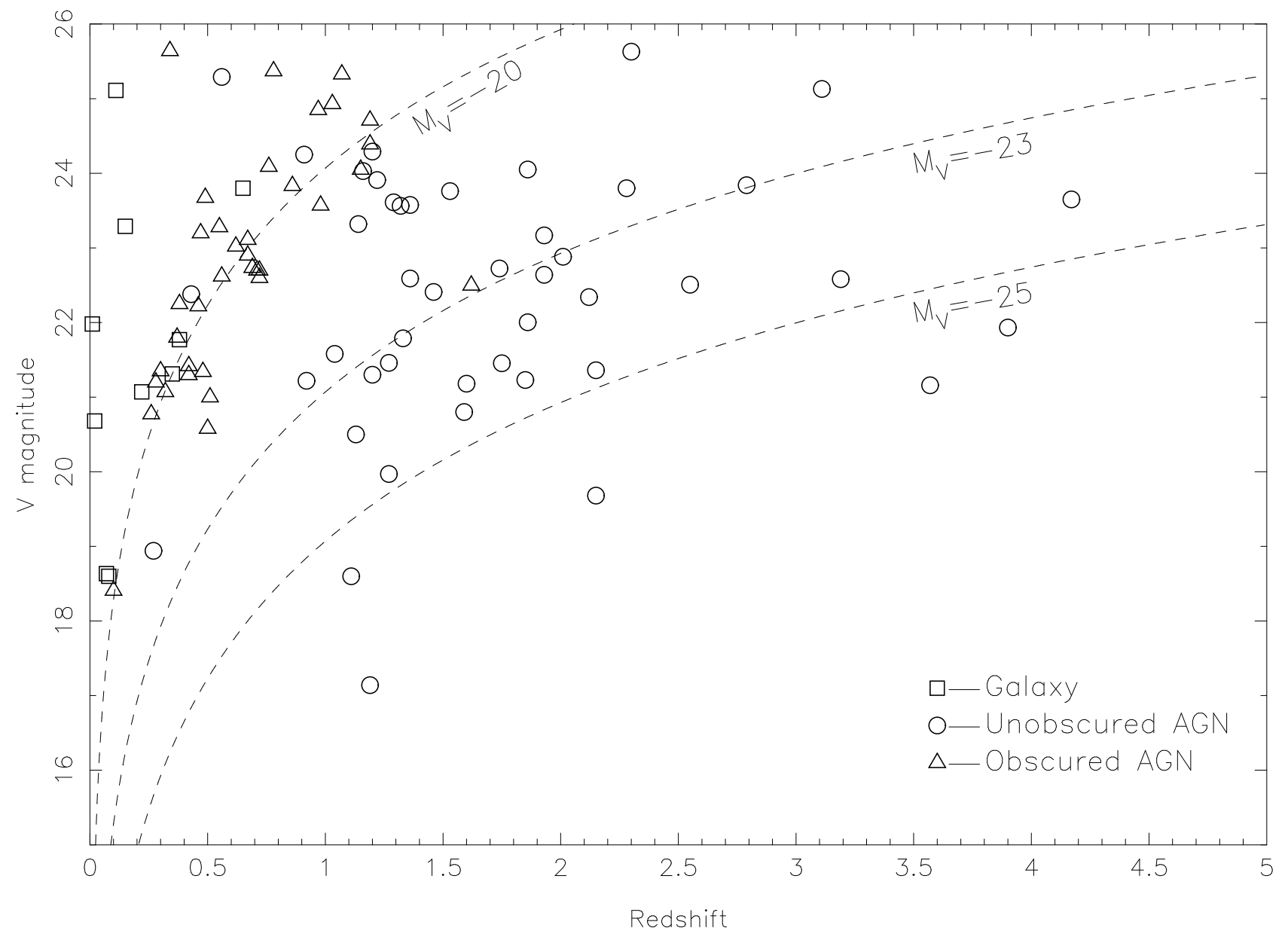

Fig. 7.- $V$-band magnitude versus redshift for sources with spectroscopic identification. Circles: unobscured (Type 1; broad lines) AGN. Triangles: obscured (Type 2; narrow lines) AGN. Squares: Galaxies. As expected, most of the high redshift sources are broad line AGN with optical magnitudes in the 22-24 mag. range. Dashed lines shows the position of constant absolute magnitude $M_{V}=-20,-23$ and -25 . While almost all the sources classified as normal galaxies or obscured AGN have $M_{V} \gtrsim-21$, unobscured (broad lines) AGN have $M_{V}<-22$. 


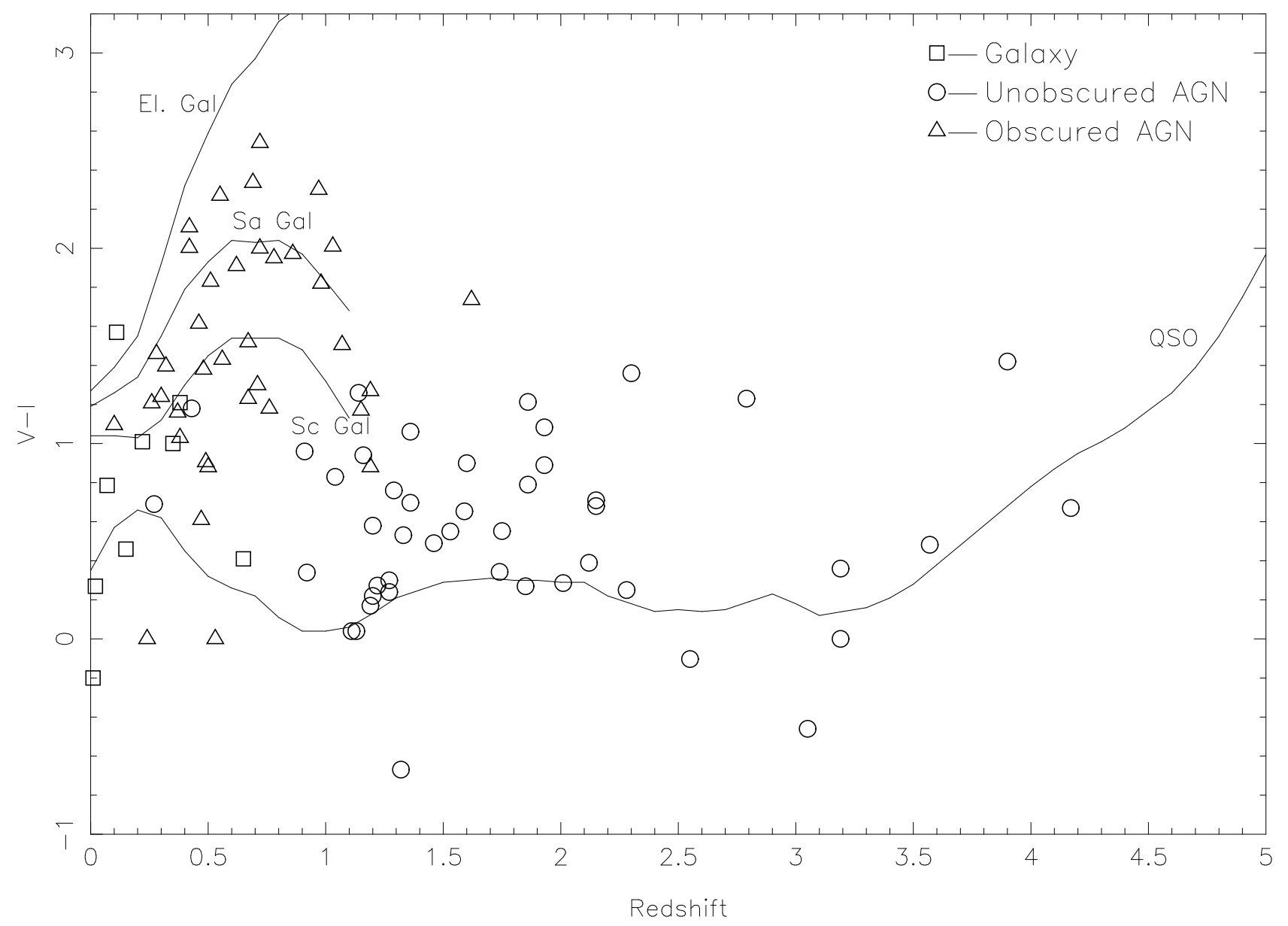

Fig. 8.- $V-I$ versus redshift for X-ray sources with spectroscopic identifications. Circles: unobscured AGN. Triangles: obscured AGN. Squares: Galaxies. Continuous lines show the expected color as a function of redshift for each type of source. Synthetic colors for galaxies were computed using the Fioc \& Rocca-Volmerange (1997) galaxy spectrum models while for the QSO track the Sloan Digital Sky Survey composite quasar spectrum (Vanden Berk et al. 2001) was used. 


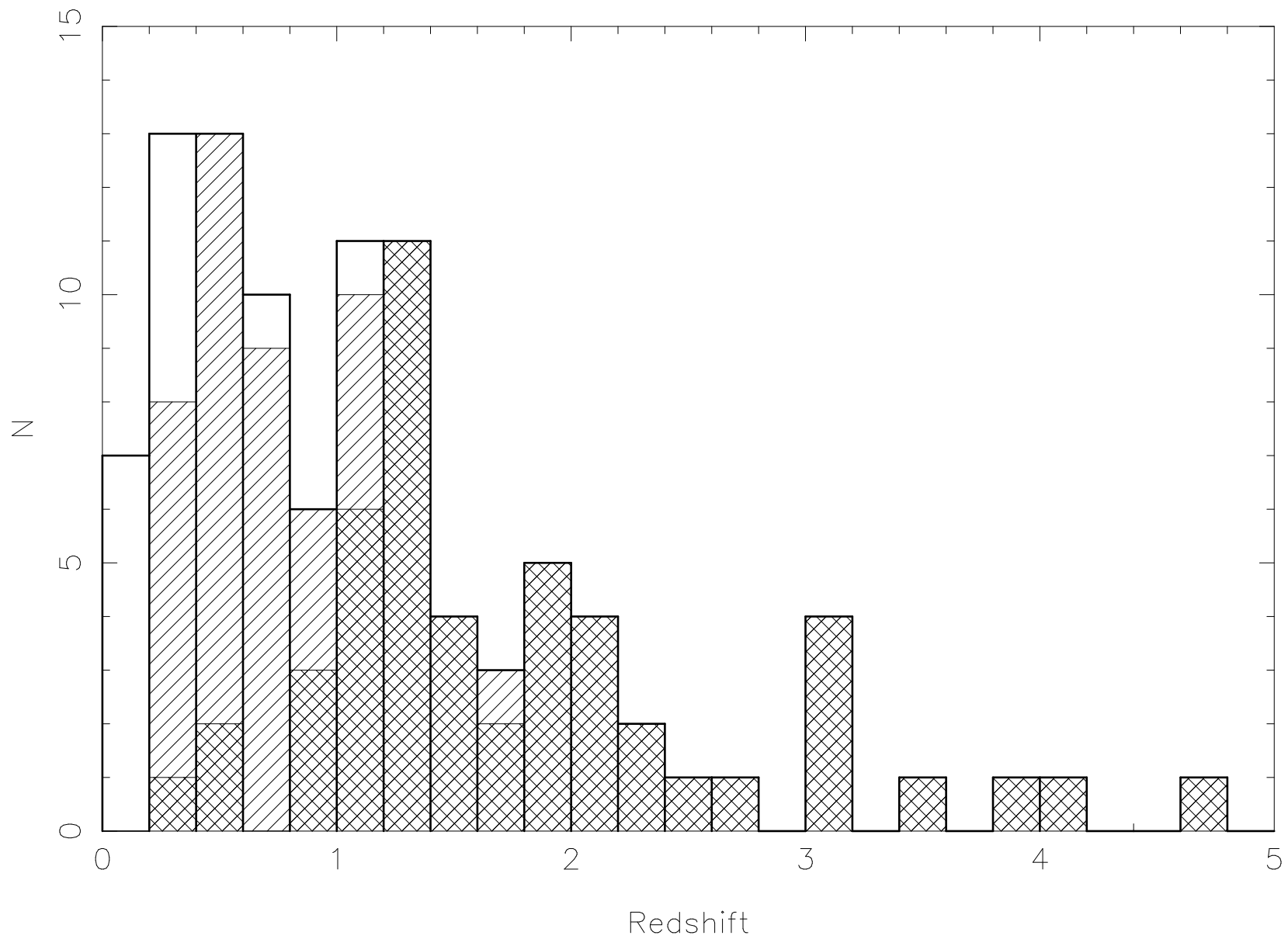

Fig. 9.- Solid line: Redshift distribution of extragalactic X-ray sources in the CYDER survey. Stars were removed for clarity. Cross-hatched histogram: redshift distribution for unobscured (broad lines) AGN, that dominate the population at high redshift $(z>1.3)$. Hatched histogram: distribution for sources with $L_{X}>10^{42} \operatorname{ergs~s}^{-1}$ (i.e., AGN dominated). In this case the broad peak of the distribution is found at $z \sim 1$. 


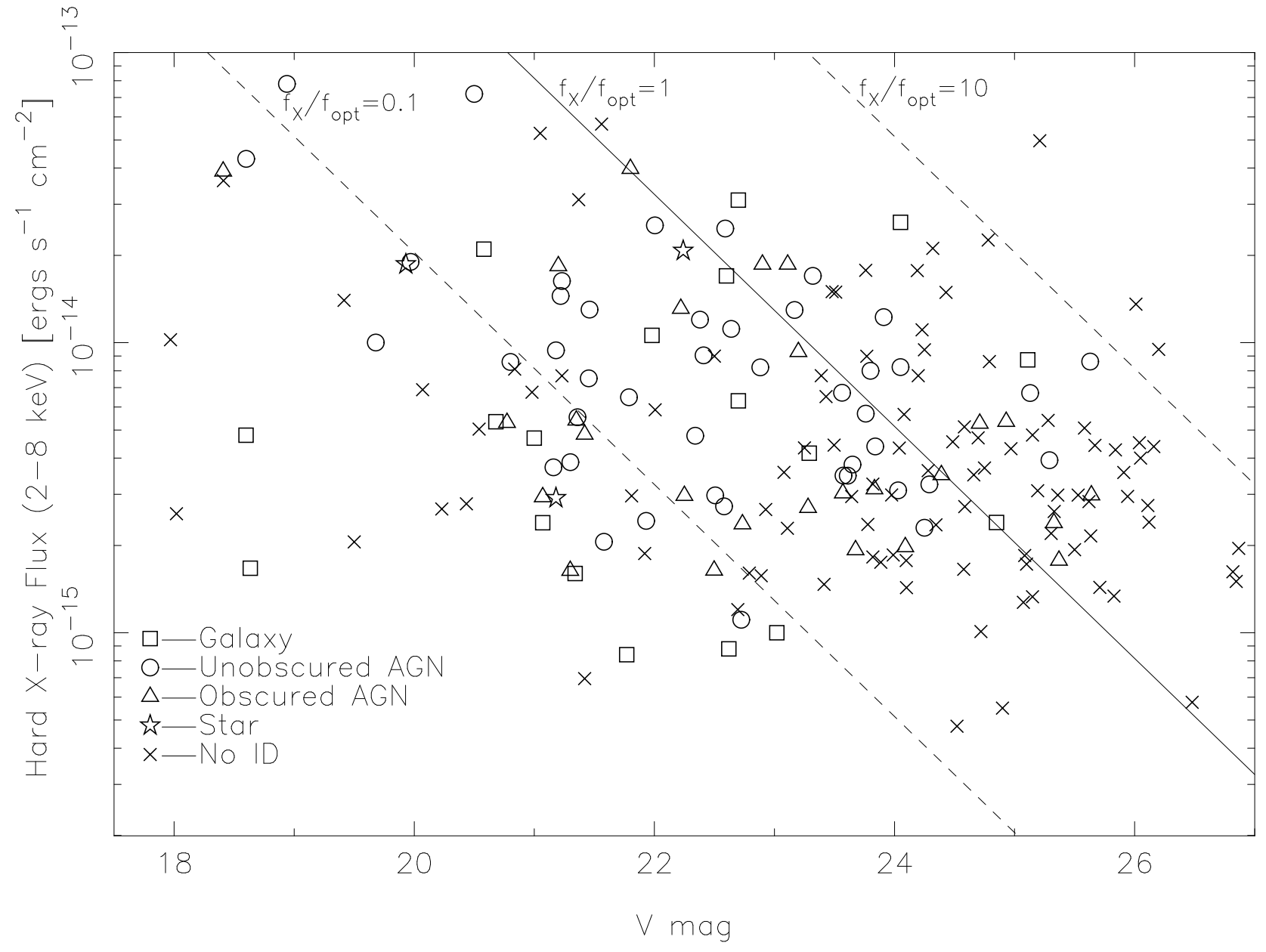

Fig. 10. - Hard (2-8 keV) X-ray flux vs $V$ band magnitude. Circles: unobscured AGN. Triangles: obscured AGN. Squares: Galaxies. Five pointed Stars: Stars with X-ray emission. Crosses: sources without spectroscopic identification. The solid line shows the locus of sources with $\log f_{X} / f_{\text {opt }}=0$, while dashed lines show the position of sources with $\log f_{X} / f_{\text {opt }}= \pm 1$. 


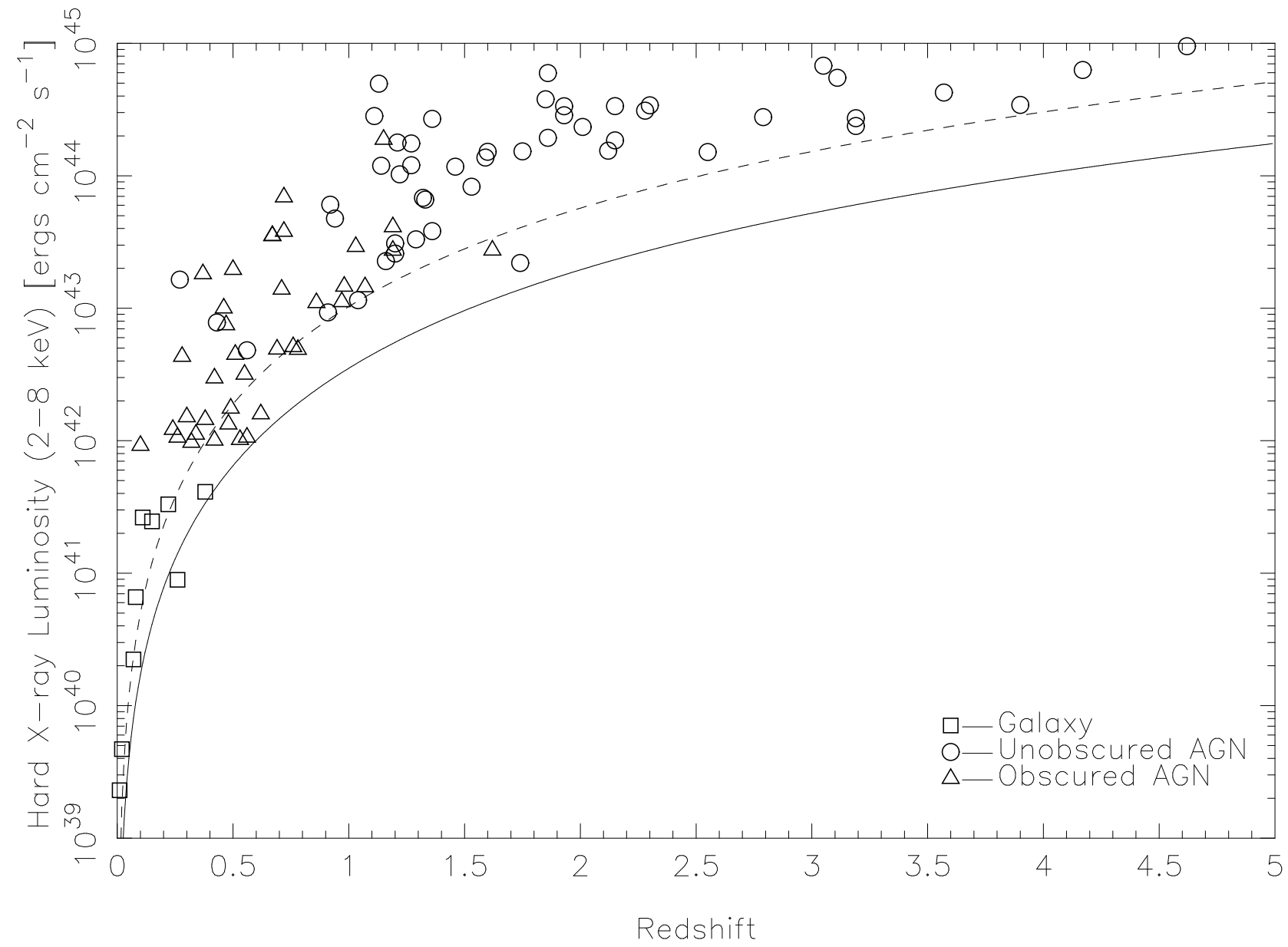

Fig. 11.- Hard (2-8 keV) X-ray luminosity versus redshift for sources with spectroscopic identification. Symbols are the same as in Figure 8. Solid line: flux limit for a simulated observation of a source detected with 5 counts in the total (0.5-8) keV band in $60 \mathrm{ks}$ with ACIS-I on board Chandra. Dashed line: X-ray luminosity for a source with optical magnitude $V=25.0$ mags and $f_{X} / f_{\text {opt }}=1$. 


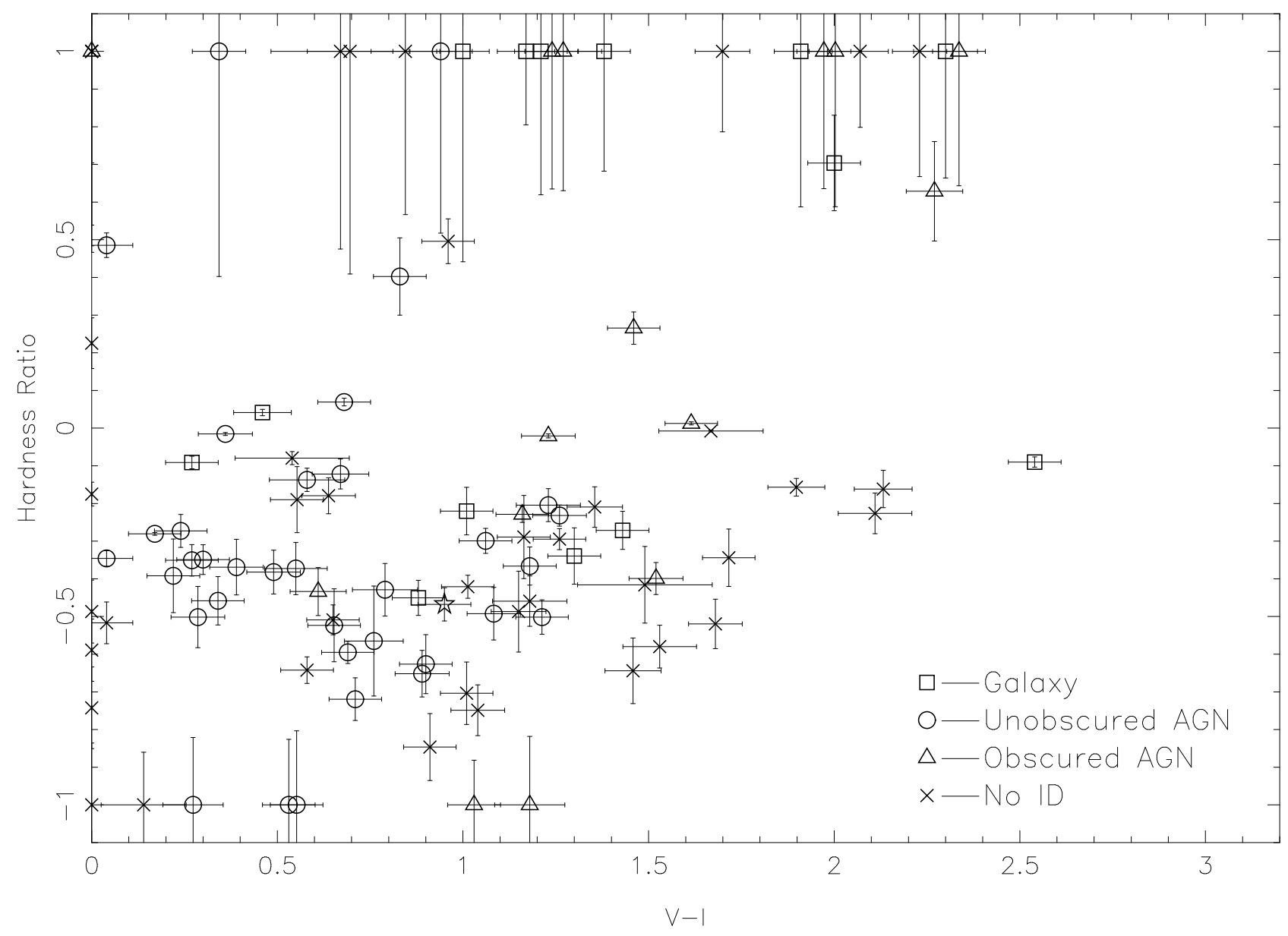

Fig. 12.- HR, defined here as $(\mathrm{H}-\mathrm{S}) /(\mathrm{H}+\mathrm{S})$ where $\mathrm{H}$ and $\mathrm{S}$ are the hard and soft X-ray band counts respectively, versus $V-I$ color; a source with $\mathrm{HR}=1$ was only detected in the hard band, while one with $\mathrm{HR}=-1$ was only detected in the soft band. Sources with fewer than 50 counts observed in the soft band and not detected in the hard band are not show in this plot. Symbols are the same as in Figure 10. Given the spread in intrinsic $V-I$ color and X-ray spectral shape, a clear correlation between HR and optical color is not observed, however a general trend can be seen in the sense that objects classified as type 2 AGN are redder and have larger HR values, consistent with the presence of obscuration affecting both X-ray and optical emission. 


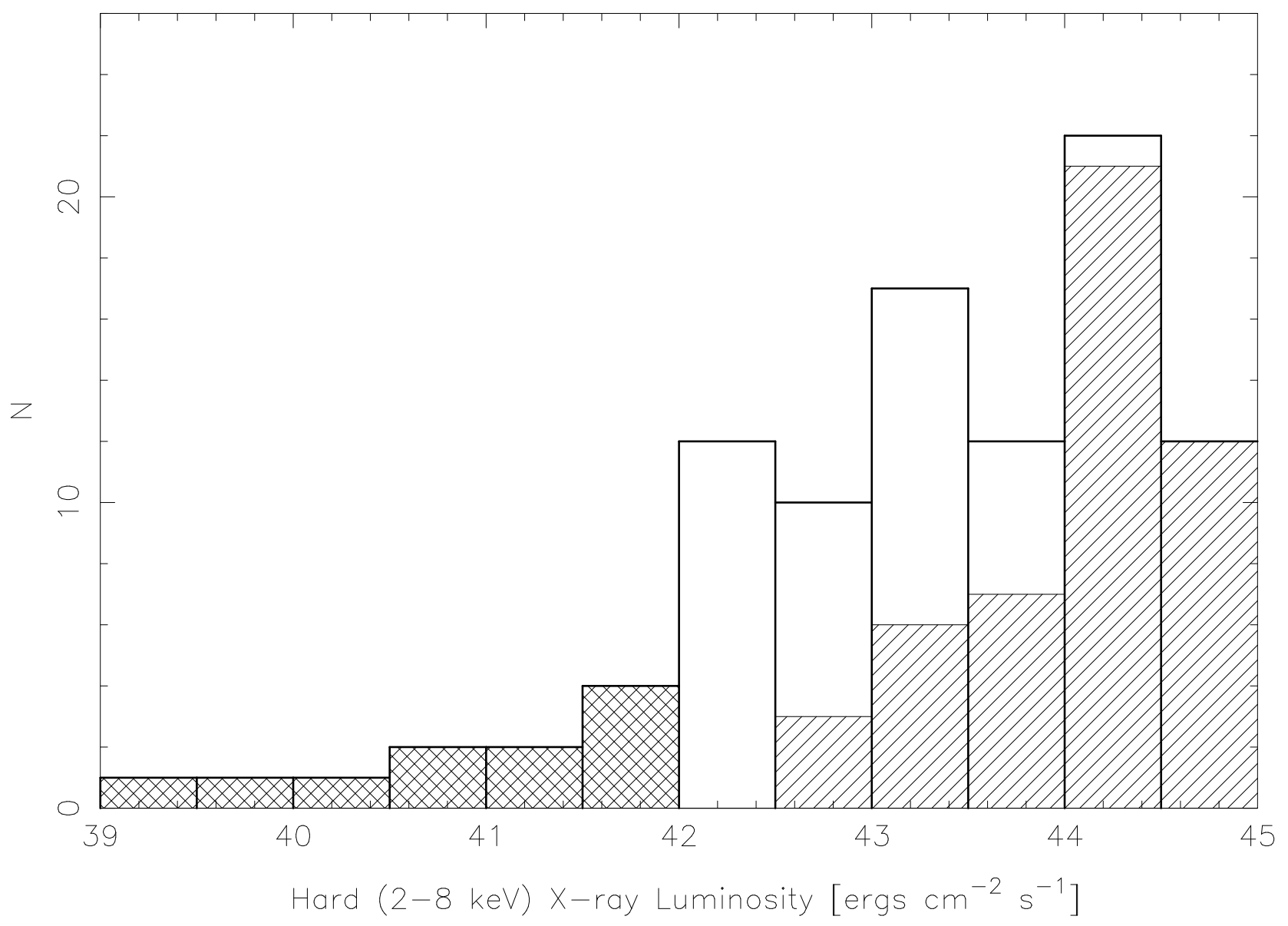

Fig. 13. - Hard (2-8 keV) luminosity distribution for the 106 sources with spectroscopic identification. Hatched histogram: luminosity distribution of unobscured AGN. Cross-hatched histogram: luminosity distribution for galaxies (i.e., $L_{X}<10^{42} \mathrm{ergs} \mathrm{s}^{-1}$ ). Unobscured AGN dominate the higher luminosity part of the distribution, while obscured AGN are the majority of the sources in the $10^{42}<L<10^{44} \mathrm{ergs} \mathrm{cm}^{-2} \mathrm{~s}^{-1}$ region. 


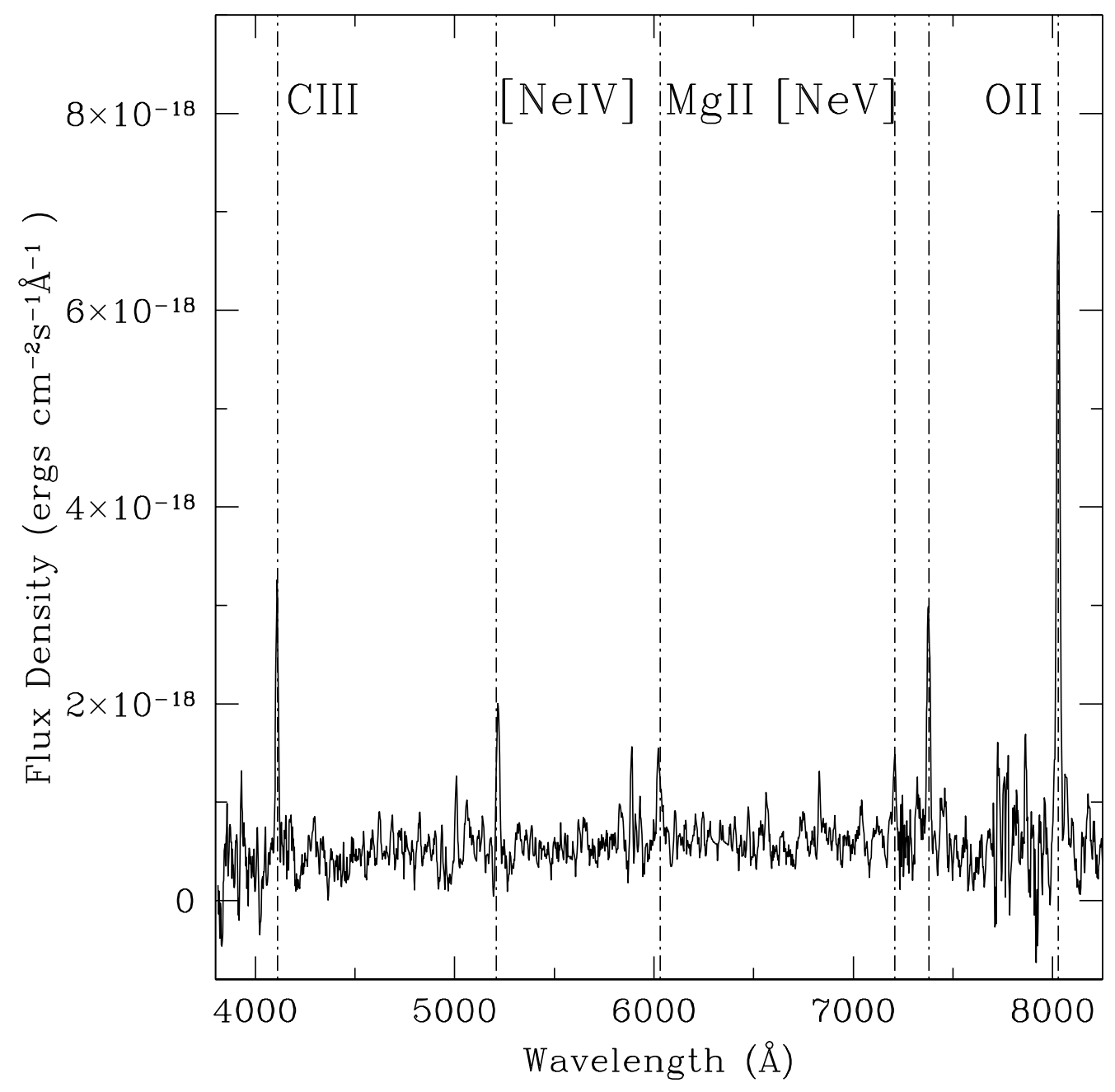

Fig. 14.- Optical spectrum of CXOCY-J125315.2-091424, the only source classified as a type 2 quasar detected in the sample. Most significant emission lines detected are identified, securing a redshift of $z=1.154$ for this source. Narrow emission lines like CIII, MgII and OII are clearly visible in the spectrum of this object. 


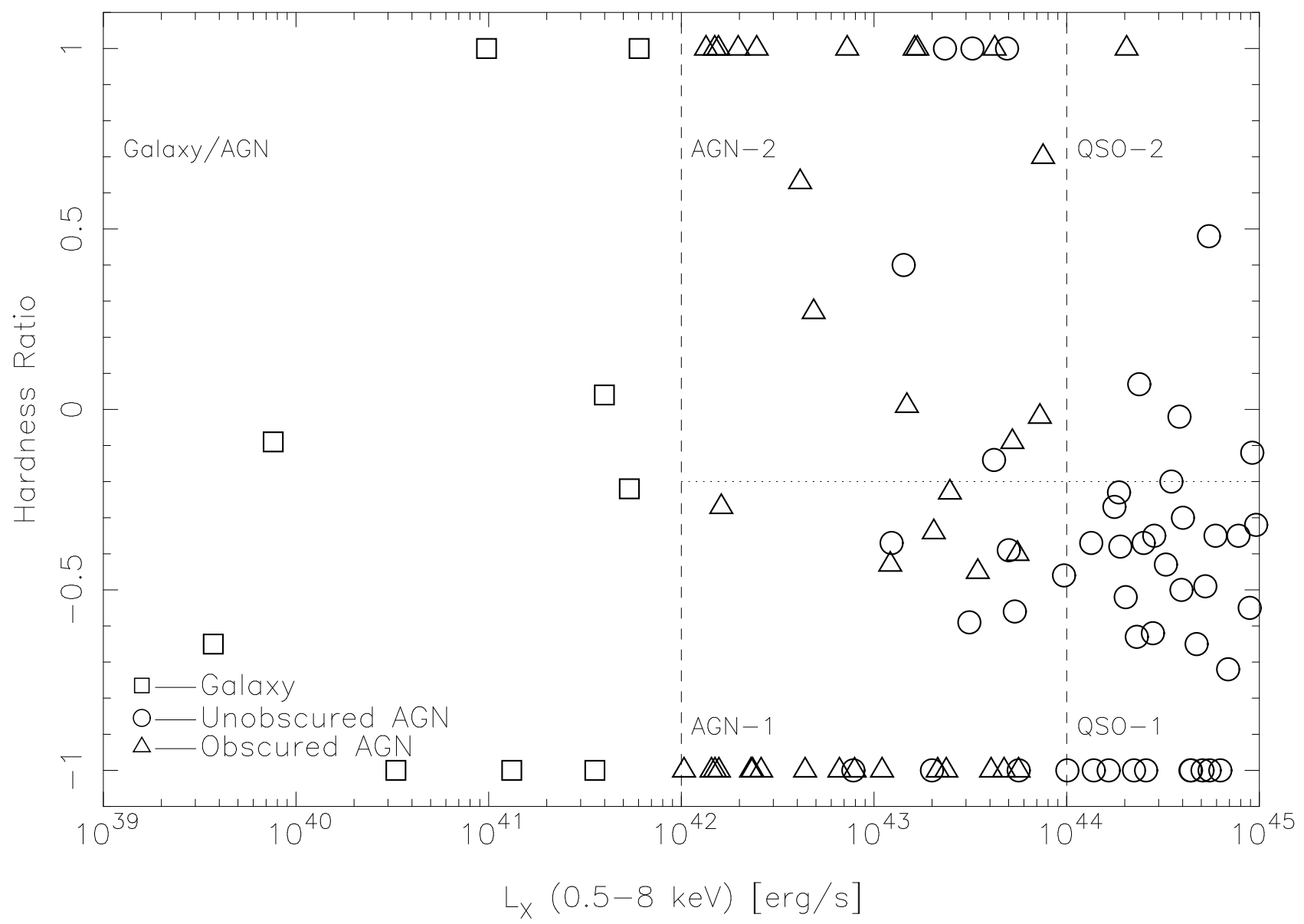

Fig. 15.- HR versus total (0.5-8 keV) X-ray luminosity. Symbols are the same as in Fig 8. Dashed lines separate galaxies and AGN at $L_{X}>10^{42} \mathrm{ergs} \mathrm{s}^{-1}$ and "Quasars" from lower luminosity AGN at $L_{X}=10^{44} \mathrm{ergs} \mathrm{s}^{-1}$. The classification scheme based on the X-ray spectral properties using $\mathrm{HR}=-0.2$ to separate obscured and unobscured AGN for sources with $L_{X}>10^{42} \mathrm{ergs} \mathrm{s}^{-1}$ (dotted line) can be compared to the scheme used in this paper based on the optical spectrum and X-ray luminosity, showing that in general obscured AGN have the X-ray hardest spectra. 


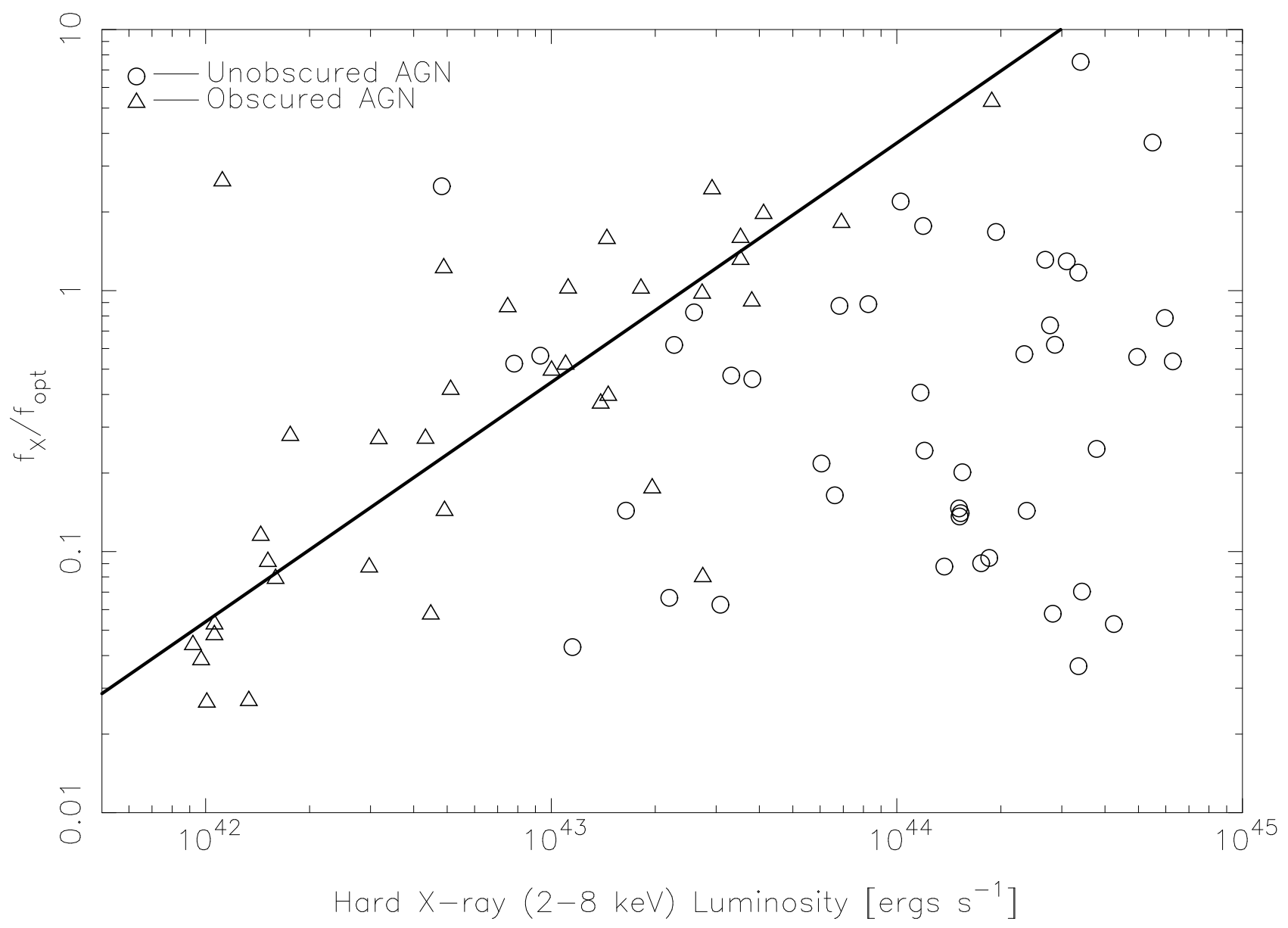

Fig. 16.- Hard X-ray to optical (measured in the observed frame $V$-band) flux ratio versus hard X-ray luminosity for sources with $L_{X}>10^{42} \operatorname{ergs~s}^{-1}$ (i.e., AGN dominated). While sources optically classified as broad line AGN (circles) are scattered over the $L_{X}>10^{42} \mathrm{ergs} \mathrm{s}^{-1}$ portion of this diagram, for obscured AGN (narrow emission lines in the spectrum; triangles) we can observe a rough correlation between $f_{X} / f_{\text {opt }}$ and $L_{X}$, namely sources with higher luminosity have larger values of $f_{X} / f_{\text {opt }}$. The solid line shows the minimum $\chi^{2}$ fit to these data. The existence of this correlation can be explained if most of the optical emission for obscured AGN comes from the host galaxy, which would be roughly independent of the luminosity of the AGN. 


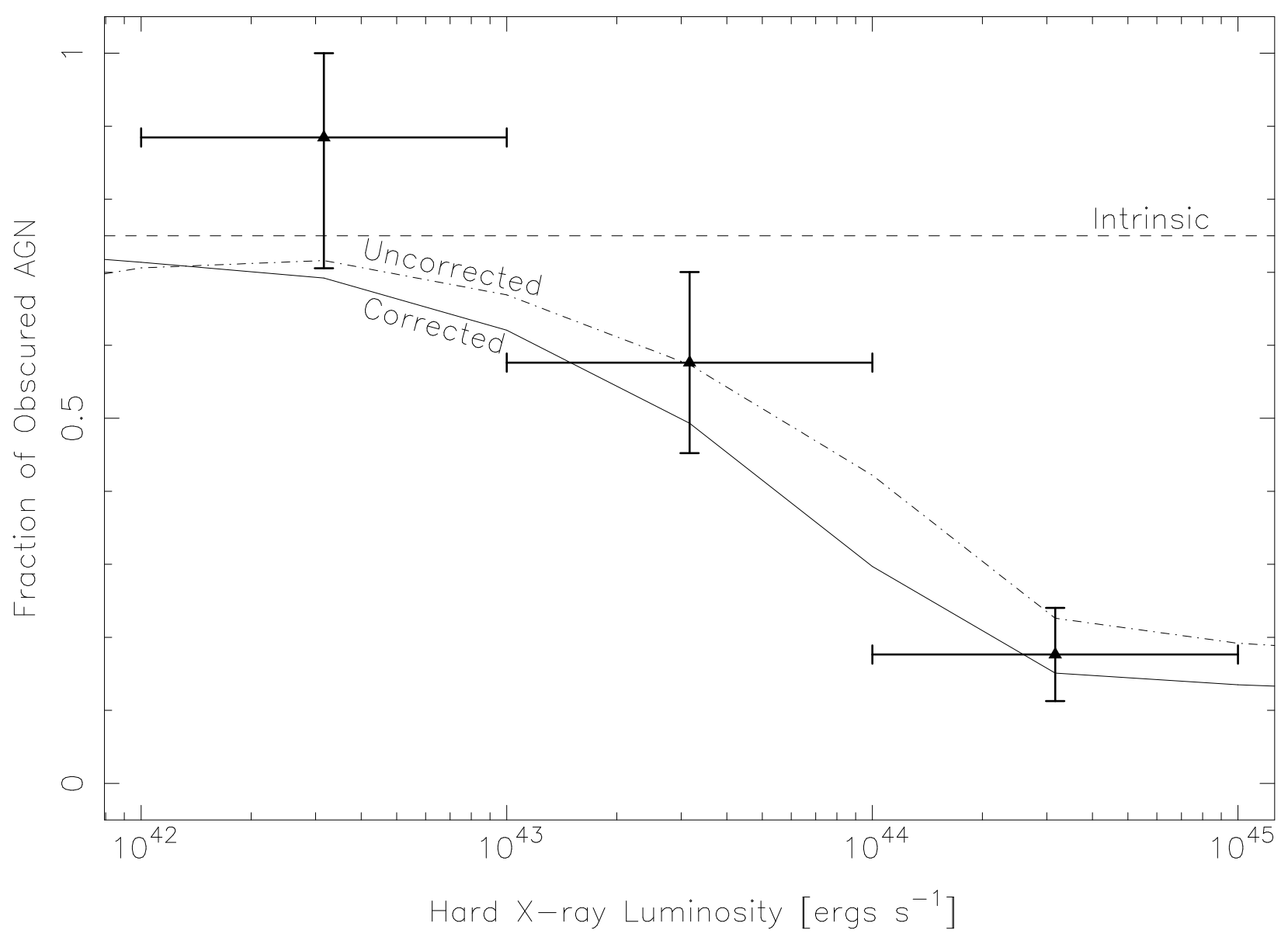

Fig. 17. - Fraction of objects optically classified as obscured AGN versus total AGN in $\Delta \log \left(L_{X}\right)=1.0$ bins combining the hard X-ray sources in the CYDER survey with the sources detected in the GOODS-S field with spectroscopic identification from Szokoly et al. (2004) in order to obtain a larger sample. The decrease in the number of obscured AGN with $\mathrm{X}$-ray luminosity can be clearly seen in this figure. (Dot-dashed line) shows the predicted correlation using the models of Treister et al. (2004b) that assumed a constant, fixed, obscured to total AGN ratio of 3:4 (dashed line) if only objects with optical magnitude $R \lesssim 24$ mag (i.e., the optical cut for spectroscopy) are considered and the effects of obscuration and k-correction are not taken into account to calculate the X-ray luminosity. Solid line shows the predicted correlation if the intrinsic hard X-ray luminosity in the model is corrected for obscuration and redshift effects. From these results, we can see that the observed correlation can be explained as a selection effect caused by the need for spectroscopic identification in order to calculate luminosities. 


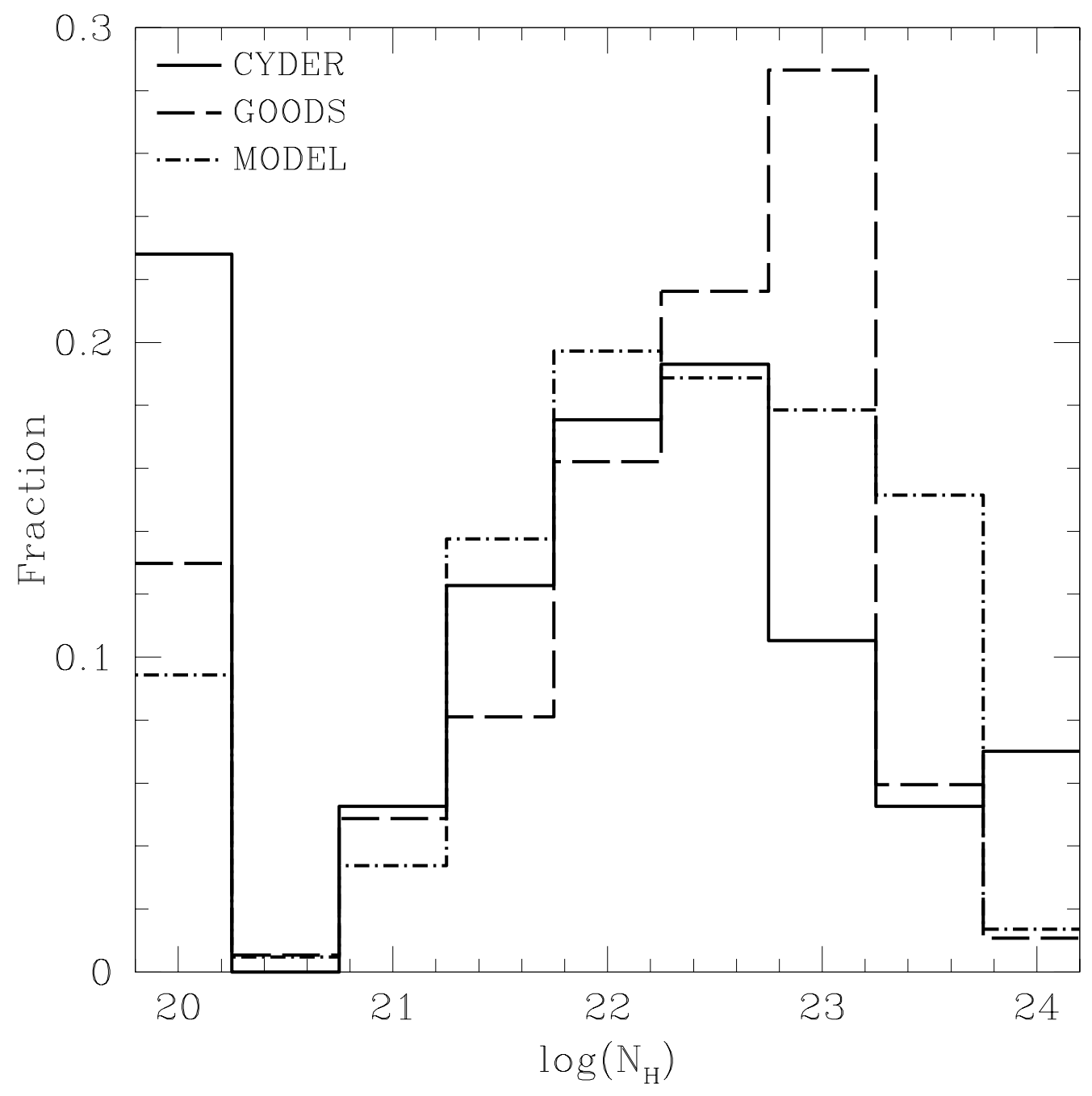

Fig. 18. - Neutral hydrogen column density $\left(N_{H}\right)$ distribution deduced for X-ray sources with measured spectroscopic redshift and detected in the hard band. The value of $N_{H}$ was calculated from the HR assuming an intrinsic power-law spectrum with exponent $\Gamma=1.9$ (solid line), typical for AGN activity, and the spectral response of the ACIS camera. The redshift of the sources was taken into account to calculate the intrinsic amount of absorption in the X-ray spectrum. The $N_{H}$ distribution for sources in the GOODS survey (dashed line), as calculated by Treister et al. (2004b) shows that in CYDER absorbed X-ray sources with $N_{H}>3 \times 10^{22} \mathrm{~cm}^{-2}$ are preferentially missed, but they appear in deeper surveys. Dotdashed line: Models from Treister et al. (2004b) adapted to the CYDER total area and flux limits. While the general agreement between can be considered good, a clear disagreement at the high $N_{H}$ end can be observed. This effect was also reported by Treister et al. (2004b) based on the GOODS data and can be explained by the incompleteness in the sample with spectroscopic redshifts (required to calculate $N_{H}$ ) since highly obscured sources are also the faintest in the optical bands. The disagreement in the low $N_{H}$ end is caused by the presence of sources with a soft-excess in the observed sample. 


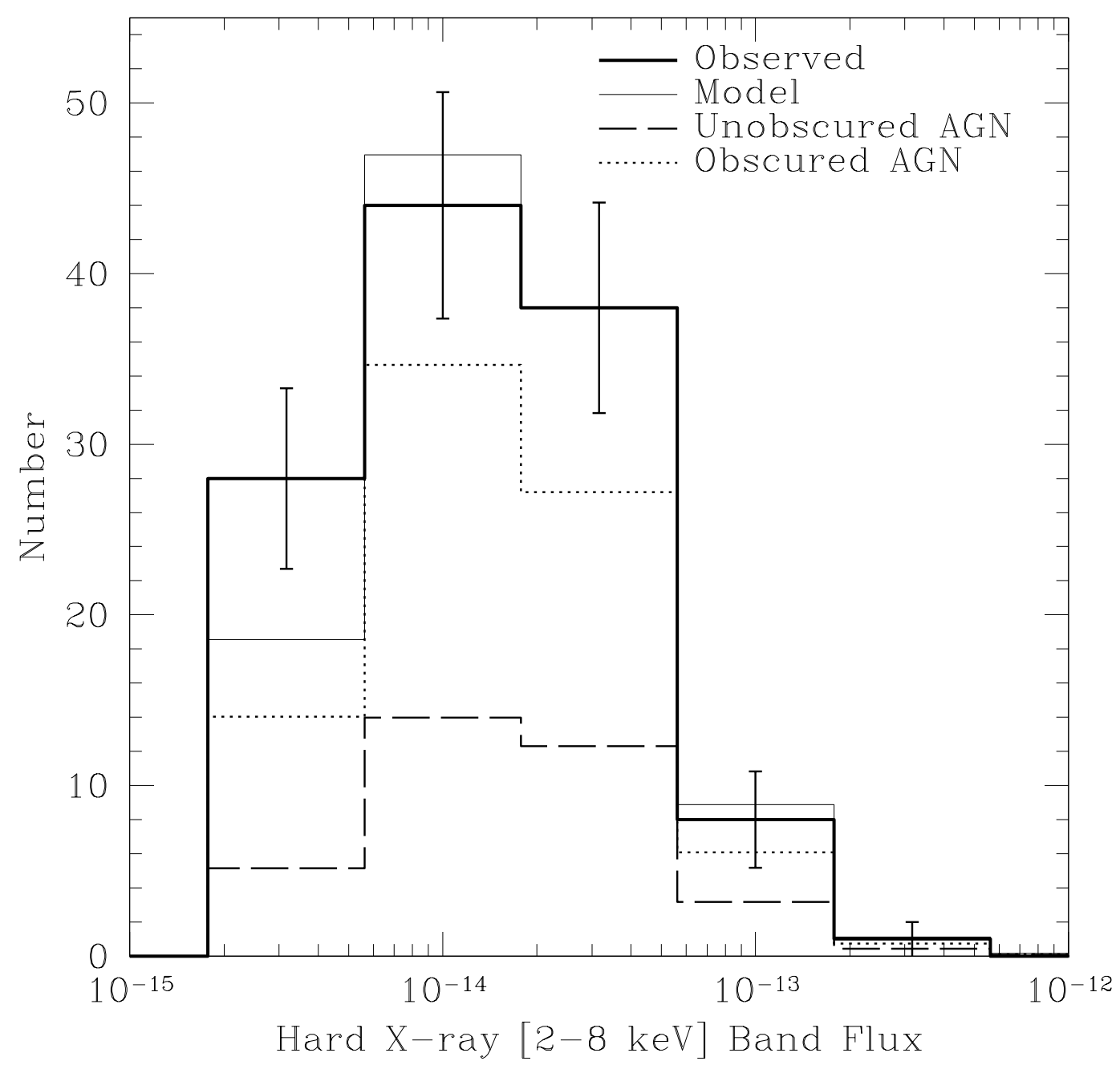

Fig. 19.- Hard X-ray (2-8 keV) flux distribution for sources detected in the CYDER fields (heavy solid line) and predicted using the simple unified models of Treister et al. (2004b); solid line. Predicted contributions by unobscured (type 1) AGN (dashed line) and obscured (type 2) AGN (dotted line) are also shown. The agreement between the predicted and observed distributions is good, with a K-S confidence level to accept the null hypothesis of $\sim 96 \%$. 